\title{
lonosphere Waves Service (IWS) - a problem-oriented tool in ionosphere and Space Weather research produced by POPDAT project
}

\author{
Csaba Ferencz ${ }^{1, *}$, Georgii Lizunov², François Crespon ${ }^{3}$, Ivan Price ${ }^{3}$, Ludmil Bankov ${ }^{4}$, Dorota Przepiórka ${ }^{5}$, \\ Klaus Brie $\beta^{6}$, Denis Dudkin ${ }^{7}$, Andrey Girenko ${ }^{8}$, Valery Korepanov ${ }^{7}$, Andrii Kuzmych ${ }^{2}$, Tetiana Skorokhod ${ }^{2}$, \\ Pencho Marinov $^{4}$, Olena Piankova ${ }^{2}$, Hanna Rothkaehl ${ }^{5}$, Tetyana Shtus ${ }^{7}$, Péter Steinbach ${ }^{1,9}$, János Lichtenberger ${ }^{1}$, \\ Arnold Sterenharz ${ }^{8}$, and Any Vassileva ${ }^{4}$ \\ 1 Eötvös Loránd University, Pázmány P.s. (1/A), 1117 Budapest, Hungary \\ ${ }^{*}$ Corresponding author: e-mail: spacerg@sas.elte.hu \\ 2 Space Research Institute NASU-SSAU, 40 Acad. Glushkova Ave., building 4/1, 03680 Kyiv, Ukraine \\ 3 NOVELTIS SAS, Rue de la Mercerie 12, 1003 Lausanne, France \\ 4 Space Research and Technology Institute BAS, Georgi Bonchev Str. bl.1., 1113 Sofia, Bulgaria \\ 5 Space Research Center PAS, Bartycka 18A, 00-716 Warshaw, Poland \\ 6 TUBerlin, Institut für Luft- und Raumfahrt, Marchstraße 12-14, 10587 Berlin, Germany \\ 7 Lviv Center of Institute of Space Research NASU-SSAU, 5-A Naukova str., 79601 Lviv, Ukraine \\ 8 ECM Office, Joachim-Karnatz-Allee 21, 10557 Berlin, Germany \\ 9 MTA-ELTE Research Group for Geology, Geophysics and Space Sci., Pázmány P.s. (1/A), 1117 Budapest, Hungary
}

Received 3 June 2013 / Accepted 10 April 2014

\begin{abstract}
In the frame of the FP7 POPDAT project the Ionosphere Waves Service (IWS) has been developed and opened for public access by ionosphere experts. IWS is forming a database, derived from archived ionospheric wave records to assist the ionosphere and Space Weather research, and to answer the following questions: How can the data of earlier ionospheric missions be reprocessed with current algorithms to gain more profitable results? How could the scientific community be provided with a new insight on wave processes that take place in the ionosphere? The answer is a specific and unique data mining service accessing a collection of topical catalogs that characterize a huge number of recorded occurrences of Whistler-like Electromagnetic Wave Phenomena, Atmosphere Gravity Waves, and Traveling Ionosphere Disturbances. IWS online service (http://popdat.cbk.waw.pl) offers end users to query optional set of predefined wave phenomena, their detailed characteristics. These were collected by target specific event detection algorithms in selected satellite records during database buildup phase. Result of performed wave processing thus represents useful information on statistical or comparative investigations of wave types, listed in a detailed catalog of ionospheric wave phenomena. The IWS provides wave event characteristics, extracted by specific software systems from data records of the selected satellite missions. The end-user can access targets by making specific searches and use statistical modules within the service in their field of interest. Therefore the IWS opens a new way in ionosphere and Space Weather research. The scientific applications covered by IWS concern beyond Space Weather also other fields like earthquake precursors, ionosphere climatology, geomagnetic storms, troposphere-ionosphere energy transfer, and trans-ionosphere link perturbations.
\end{abstract}

Key words. ionosphere (general) - wave - signal processing - density - services

\section{Introduction}

Satellite records form the primary base for studying processes in terrestrial plasma (ionosphere, magnetosphere) which have a key importance in geophysical and Space Weather research and monitoring. Despite the large number of satellite missions successfully operated in the Earth environment, only fewer missions provide wave records, especially related to the ionosphere, and these recorded data sets did not form a consistent unified data base or time series of interesting phenomena.

Public ionosphere satellite data sources (e.g. http:// sevem.aeronomie.be, http://spidr.ngdc.noaa.gov/spidr/) typically provide optional query capabilities, according to missions, instrument, data type, time window, resulting in a set of raw or level 1 processed recordings. Consequently the task of selection or detection of certain phenomena, e.g. specific waves is supposed to be performed at the end-user side.

According to the complexity of coupled, parallel physical processes in the ionosphere it is useful and reasonable to handle investigated phenomena, e.g. waves in a way that supports comparative studies, starting already at high level, user-oriented processed data. This report summarizes the concept and first results of a new, public ionosphere wave analysis tool, built and started in frame of the completed EU FP7 POPDAT program.

\section{Outline of the IWS concept}

In the near past satellite experiments were addressed to study the main morphologic features and global distribution of the 
Table 1. Main ionosphere satellite missions potentially relevant in the creation of the IWS by their particle data and wave recording.

\begin{tabular}{|c|c|c|c|c|}
\hline No & Mission & Year & Orbit & Payload \\
\hline 1 & $\begin{array}{l}\text { Atmosphere } \\
\text { Explorer-A }\end{array}$ & $1963-1966$ & $255-916 \mathrm{~km}, i=57^{\circ}$ & $\begin{array}{l}\text { Sensors for neutral and ionized species of atmospheric } \\
\text { gazes. }\end{array}$ \\
\hline 2 & $\begin{array}{l}\text { Atmosphere } \\
\text { Explorer-B }\end{array}$ & $1966-1967$ & $276-2725 \mathrm{~km}, i=64^{\circ}$ & $\begin{array}{l}\text { Sensors for neutral and ionized species of atmospheric } \\
\text { gazes. }\end{array}$ \\
\hline 3 & $\begin{array}{l}\text { Atmosphere } \\
\text { Explorer-C }\end{array}$ & $1973-1978$ & $149-4294 \mathrm{~km}, i=68^{\circ}$ & $\begin{array}{l}\text { Sensors for neutral and ionized species of atmospheric } \\
\text { gazes, optical and UV spectrometers. }\end{array}$ \\
\hline 4 & $\begin{array}{l}\text { Atmosphere } \\
\text { Explorer-D }\end{array}$ & $1975-1976$ & $154-3816 \mathrm{~km}, i=90^{\circ}$ & $\begin{array}{l}\text { Sensors for neutral and ionized species of atmospheric } \\
\text { gazes. }\end{array}$ \\
\hline 5 & $\begin{array}{l}\text { Atmosphere } \\
\text { Explorer-E }\end{array}$ & $1975-1976$ & $156-2983 \mathrm{~km}, i=20^{\circ}$ & $\begin{array}{l}\text { Sensors for neutral and ionized species of atmospheric } \\
\text { gazes. }\end{array}$ \\
\hline 6 & $\begin{array}{l}\text { Dynamics } \\
\text { Explorer } 2\end{array}$ & $1981-1983$ & $309-1012 \mathrm{~km}, i=90^{\circ}$ & $\begin{array}{l}\text { Sensors for neutral and ionized species of atmospheric } \\
\text { gazes, sensors for quasi DC magnetic field, DC and AC } \\
\text { electric field. }\end{array}$ \\
\hline 7 & San Marco D/L & $1988-1988$ & $262-619 \mathrm{~km}, i=2^{\circ}$ & Neutral wind probe, plasma probes, electric field probe. \\
\hline 8 & $\begin{array}{l}\text { Active } \\
\text { (Intercosmos-24) }\end{array}$ & $1989-1993$ & $500-2500 \mathrm{~km}, i=82^{\circ}$ & $\begin{array}{l}\text { Plasma probes, sensors for quasi DC electric and } \\
\text { magnetic fields, wave probes, energetic particle probe, } \\
\text { VLF-transmitter. }\end{array}$ \\
\hline 9 & APEX & 1991 & $440-3000 \mathrm{~km}, i=82^{\circ}$ & $\begin{array}{l}\text { Sensors for neutral and ionized species of atmospheric } \\
\text { gazes, electric beam and plasma injectors. }\end{array}$ \\
\hline 10 & FREJA & 1992 & $650-1800 \mathrm{~km}, i=65^{\circ}$ & $\begin{array}{l}\text { Plasma probes, high data rate electromagnetic wave } \\
\text { probes, energetic particle probes, UV imager. }\end{array}$ \\
\hline 11 & GPS system & 1994 & $20,200 \mathrm{~km}, i$ different, $\geq 24$ satellite & For ionosphere research: total electron content (TEC). \\
\hline 12 & ROCSat-1 & 1999-2004 & $600 \mathrm{~km}, i=35^{\circ}$ & Plasma probes. \\
\hline 13 & CHAMP & $2000-2010$ & $454 \mathrm{~km}, i=87^{\circ}$ & Magnetic field probes, precise accelerometer. \\
\hline 14 & $\begin{array}{l}\text { Variant } \\
\text { (on Sich-1M S/C) }\end{array}$ & 2004-2005 & $280-650 \mathrm{~km}, i=83^{\circ}$ & $\begin{array}{l}\text { Electric probes, electromagnetic wave probes, electric } \\
\text { current probes, fluxgate magnetometer. }\end{array}$ \\
\hline 15 & DEMETER & 2004-2010 & $\begin{array}{c}\sim 710 \mathrm{~km}, \text { changed to } 660 \mathrm{~km}, \\
i=98^{\circ}\end{array}$ & $\begin{array}{l}\text { Plasma probes, electric field and } \mathrm{AC} \text { magnetic field } \\
\text { probes. }\end{array}$ \\
\hline 16 & Compass-2 & $2006-2007$ & $\sim 400 \mathrm{~km}, i=79^{\circ}$ & Electromagnetic wave probes in ULF-VLF bands. \\
\hline
\end{tabular}

atmospheric and ionospheric parameters (altitudinal, latitudinal, diurnal, etc.). Large amount of archived satellite data gathered during these earlier missions are successfully integrated in various models of the upper atmosphere and ionosphere. Upcoming data from the current missions have been used for the adjustment of existing models or for the development of alternative models. During this long research work a lot of transient, propagating wave-like phenomena were identified. The need of regular investigating such ionospheric phenomena is obvious. However, the effectiveness of the systematic investigation of these phenomena is much limited using raw or 1st level processed satellite recordings, due to their typically huge occurrence number and variable signal characteristics.

At present, several new techniques are available in ionospheric data evaluation, providing the chance to assemble an integrated database, containing characteristics of different kind of ionospheric phenomena, categorized by type, by satellite mission, covering a longer time period. New data mining techniques form the base of the idea of the Ionosphere Waves Service (IWS). IWS, serving most important derivatives and characteristics of wave phenomena, with its higher level processing is thought to represent new approach of ionospheric research. In this new way the operator or end-user is provided by a processed data, searching for a specific physical phenomenon by querying the database and let predefined recognition process to run during data collection procedures.

Processing the data of the satellite experiments includes some important levels: the level 1 is the raw data conversion, decoding of the control parameters, removing erroneous data records, adjustment to the universal time, etc. This process transforms the telemetry data flow to the basic electrical signals relevant to the specific application of the onboard instrumentation as a chain of equidistant records in time. At the level 2, these records of electrical values are transformed to the real physical one by the use of the next level (calibration, etc.) algorithms quite specific for different experiments.

The idea of the IWS has been to apply third level data processing, using level 2 data as input, and with this step creating a new, unified database: the goal to achieve in the EU FP7 POPDAT project (see more at the project official website at: http:// popdat.org). The main purpose of this level 3 processing is to offer an opportunity to the user to select specific wave categories (listed in Sect. 5) from the processed satellite data set which corresponds to the intriguing physical phenomena, by use of specifically designed software tools. Since the wave-like phenomena are one of the most informative "tools" in investigating the ionosphere and huge variety of ionosphere related physical processes (e.g. atmosphere-ionosphere coupling, including possible seismo-electromagnetic effects, emissions due to wave-particle interactions), the IWS database has been selected to include 3rd level processed waves, in general transients. These wave-like phenomena cover a wide range of known phenomena in the plasma, from propagating VLF signals to ion density variations at different scales, or "double layer" structures in quasi DC electric field, etc. This level 3 of data processing requires a priori knowledge about the selected wave type to be processed, and the specific algorithm to be adjusted accordingly. Data processing covers here mass wave recognition, detection, and first-order automatic derivation of wave characteristics in level 1 and 2 data sets, detailed in Section 3. Besides date, time, and satellite location, set of event specific parameters are determined and stored during the processing. Interested reader (potential user) finds detailed description on IWS database items, query options at the service 
(a)

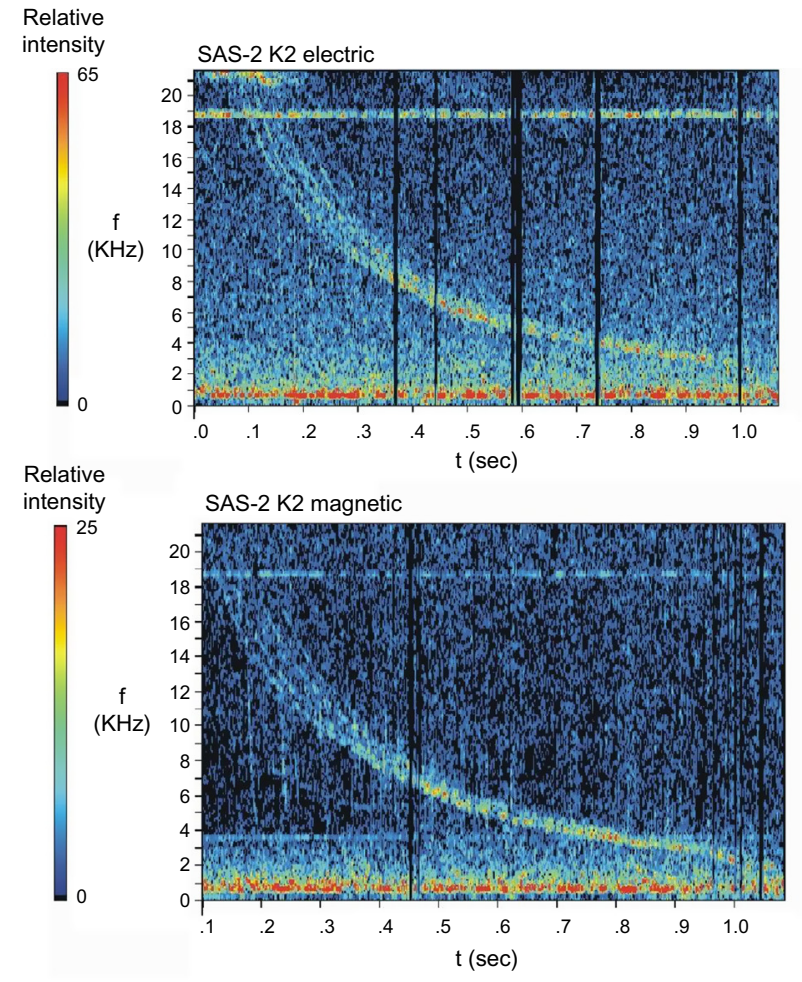

(b)
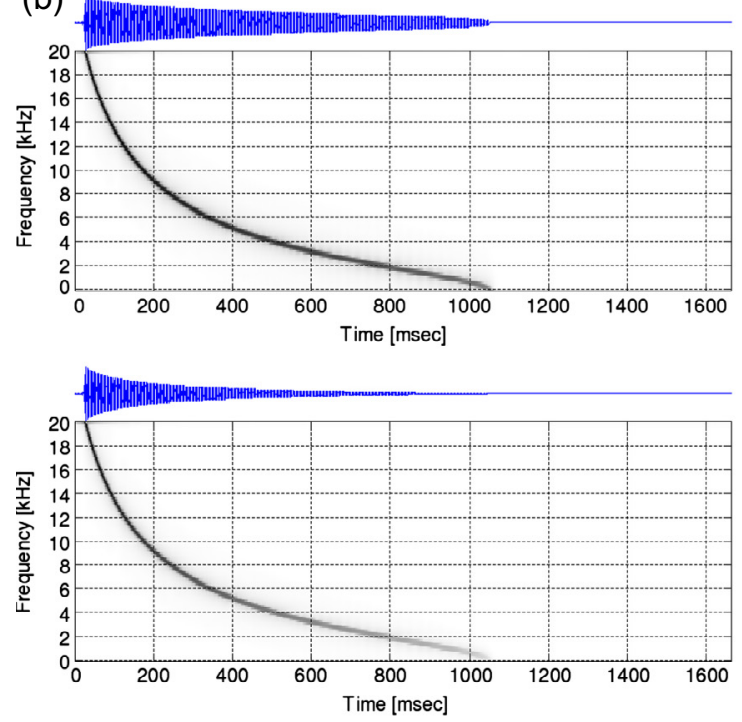

(c)
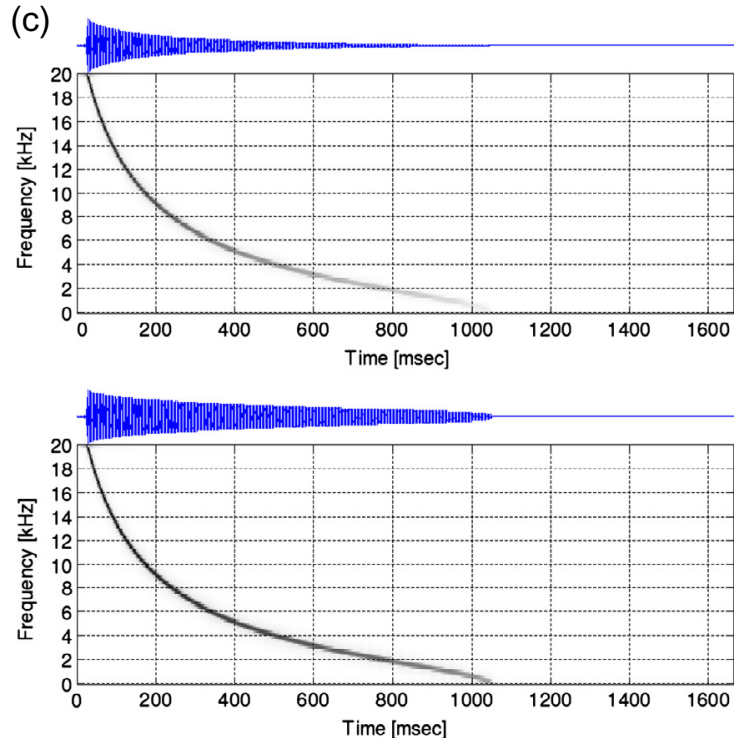

Fig. 1. (a) FFT pattern of a ducted whistler, measured on board of Compass-2 satellite on 28.02.2007 07:32 UT by the SAS2-K2 instrument. Logarithmic power spectrum of electric and magnetic field components are displayed in relative scale, (b) the FFT patterns of the computed (UWB modeled) theoretical whistler components, propagating between two layers of inhomogeneities, the $E_{y}$ (upper panel) and $E_{z}$ (lower panel) components, and (c) the $H_{y}$ (upper panel) and $H_{z}$ (lower panel) components. The $y$ direction is orthogonal to the direction of propagation and to the layers of inhomogeneities; the $z$ direction is orthogonal to the direction of propagation and parallel with the layers of inhomogeneities. Above the FFT pattern of the modeled UWB signals the computed time functions of the modeled field strengths are presented (Ferencz et al. 2009).

website (http://popdat.cbk.waw.pl). The current level of information technologies reveal realistic opportunities, level 3 data mining to be used over sets of data taken from different satellites at different places and times, combined with sort of solar-geophysical data.

The aim of the IWS is not that it would include any new kind or set of measured data, but serving purely the derived parameters of described wave-like phenomena, obtained by specific algorithms. All processed data are archived, stem from not operational missions. IWS assists to gather and serve new information out of them, especially for comparative/statistical studies.

IWS realized according to the above concept may assist various investigations in Earth science and in Space Weather research, and being public, opens the way for large number of researcher - not necessarily involved in the specific satellite project teams, working groups, etc., but interested in effective data mining and interpretation. 

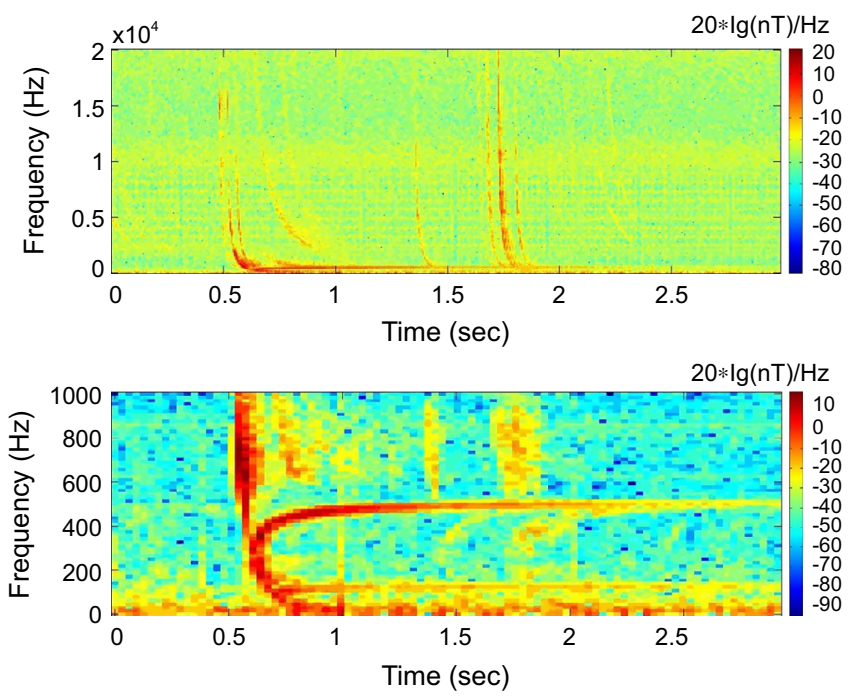

Fig. 2. The FFT dynamic spectrum of a DEMETER IMSC magnetic field record section (25.09.2004 21:29:20 UT). The upper picture is the VLF pattern of the record and the lower picture is the ELF band of same record. In the upper picture several fractional hop whistlers appeared and accompanied to the first fractional-hop $\left(0^{+}\right)$whistler appeared the more dispersed second trace of a trapezoid whistler pair. In the lower picture (proton and helium) ion whistlers are also seen where the proton whistler has the higher and the helium whistler has the lower upper frequency asymptote, according to $\mathrm{H} / \mathrm{He}$ gyro-frequencies.

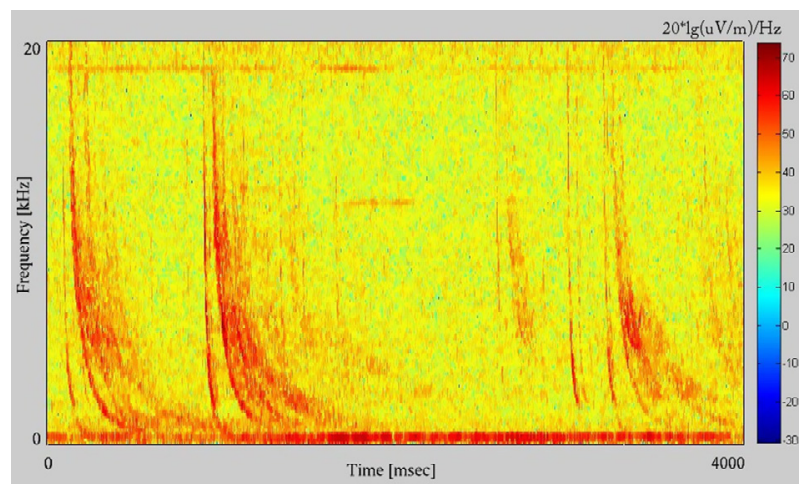

Fig. 3. The FFT dynamic spectrum of a low-latitude whistler pair series, measured on board of DEMETER satellite, ICE VLF burst data (20.08.2004 13:54:32 UT).

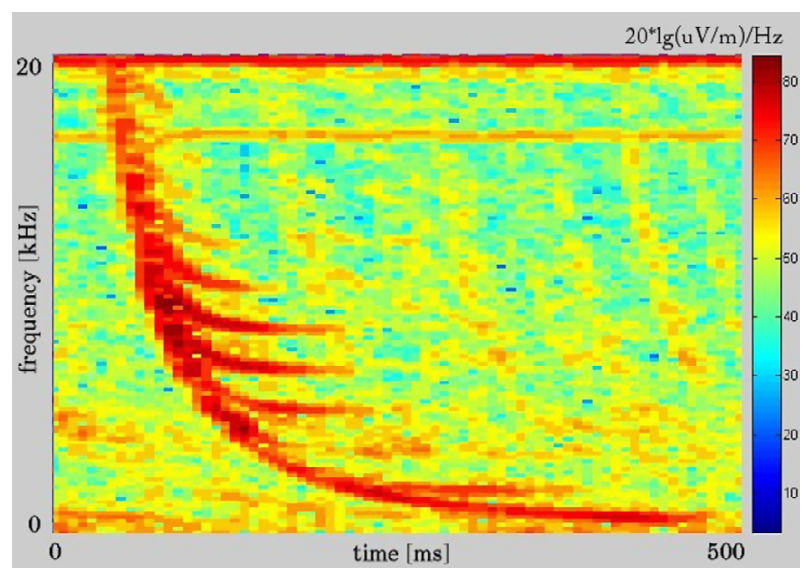

Fig. 4. The pattern of a spiky whistler (SpW) in the FFT spectrum, recorded on board of DEMETER satellite, ICE VLF burst data (25.09.2004 21:30:31 UT).

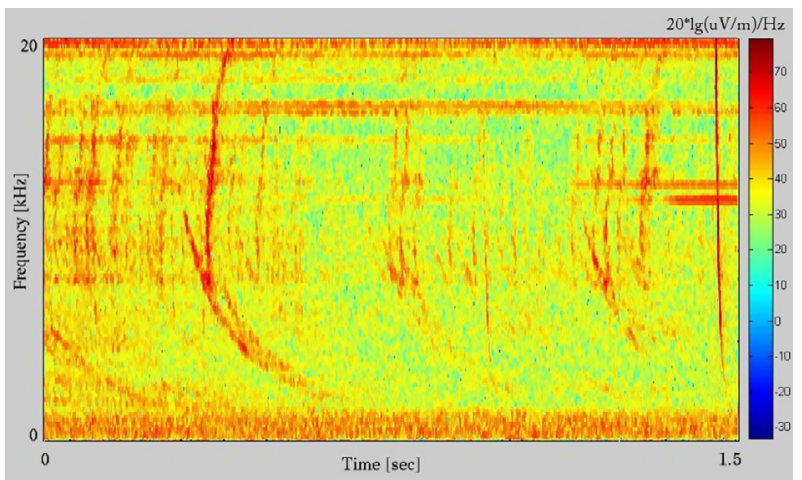

Fig. 5. The pattern of STW signals in the FFT spectrum, recorded by the DEMETER satellite, ICE VLF burst data (16.10.2004 20:55:16 UT).

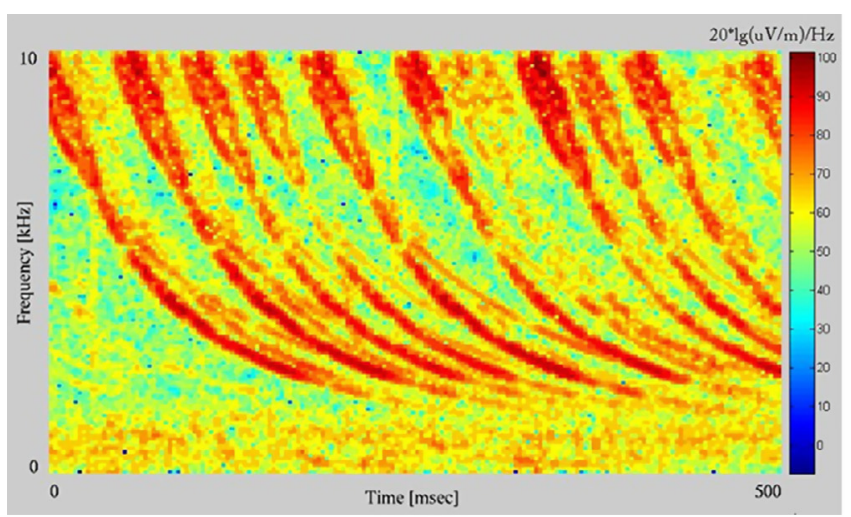

(a)

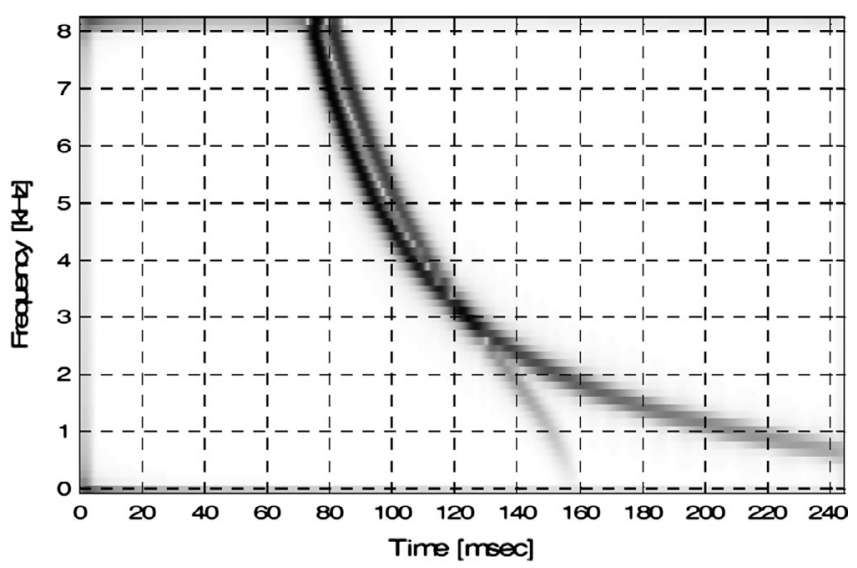

(b)

Fig. 6. (a) One type of crossing traced, X-shaped whistler or whistler-like signal branch recorded on board of DEMETER satellite, ICE VLF burst data (20.09.2004 21:03:20 UT). (b) Theoretical UWB propagating model in which the propagating modes are guided in a duct and in the FFT pattern are crossing each other (courtesy, O.E. Ferencz).

\section{Satellite missions interesting in ionosphere investigations}

Since the beginning of the satellite investigations of the Earth and Earth's environment more than 20 satellites were launched for the investigation of the ionosphere. Table 1 contains the list of a subset of satellites, providing direct waveform records and/ or particle data, which could be involved for the investigations 


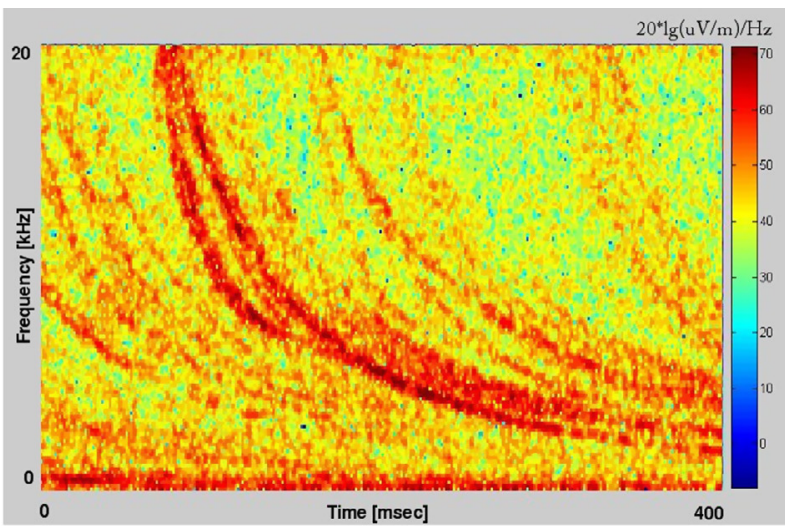

(a)

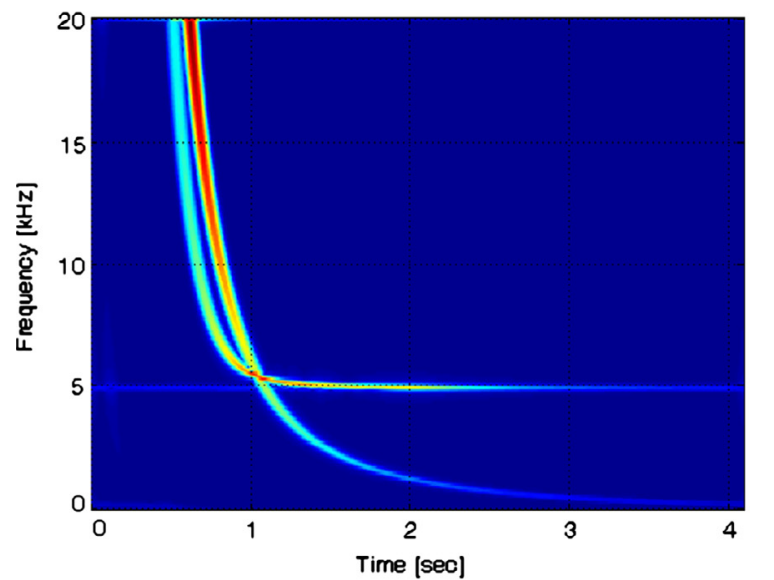

(b)

Fig. 7. (a) Another type of crossing traced, $X$-shaped signal with slightly different character recorded on board of DEMETER satellite, ICE VLF burst data (13.10.2004 03:26:40 UT). (b) FFT pattern of theoretically computed signals where one signal part propagated in first guided mode in the Earth-ionosphere waveguide and after this crossed the ionosphere, and the other signal propagated in a direct path from the same lightning to the satellite in oblique direction comparing to the geomagnetic field-lines (courtesy, O.E. Ferencz).

of transient phenomena in the ionosphere and therefore it was necessary to investigate the possible application of the recorded data sets of these missions during the creation of the IWS.

During the creation of the IWS the first step was a critical review of this list of satellites to select the most appropriate satellite missions in data base buildup point of view. The broadening of the satellite list in the future after the start of the operation of the IWS is already possible, however, in the first step we must have used only the fully available data sets. This means that the incorporation the data of new satellite missions which are in operation at this time or will start in the near future is also possible.

The selection criteria of a satellite mission, preferable for the creation of the IWS are the following:

In general the accessibility and availability of recorded data sets was one important criterion. For example the APEX (USSR) mission was a considerable space project. However, the data recorded and transmitted to the Earth are not in digital electronic form in an open access database. Therefore it was not possible to include this satellite into the creation phase of the IWS. Open (e.g. internet) access to the recorded data, the

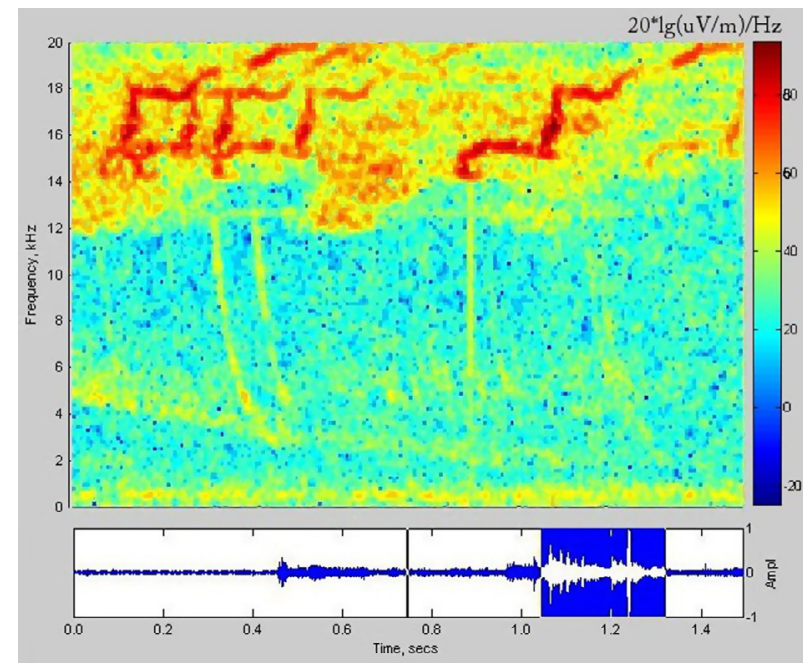

Fig. 8. A series of signals with strange shape recorded on board of DEMETER satellite, ICE VLF burst data (29.09.2004 10:58.05 UT). These signals in this case are accompanied or appearing simultaneously with whistlers and fractional hop whistlers.

original "raw" data of a given satellite was very important in selection a satellite during this first creation step.

Next, further selection criteria were applied in the case of electromagnetic wave (EMW) data. These are the following: according to the orbit the recorded electromagnetic signal is ionospheric one $(\leq 800-900 \mathrm{~km}$ in the moment of or during the measurement) and it has no "magnetospheric" character. It is important, that the time domain waveform is present, the data is real broadband ELF-VLF "burst"-type data in digital electronic form with the required accuracy, i.e. the preferred recorded frequency band is $>50-100 \mathrm{~Hz}$ to $\leq 20-40 \mathrm{kHz}$. In the case of older missions the $5 \mathrm{kHz}$ upper frequency limit was also accepted. The required accuracy was the minimum 8 bit resolution of a sample, but the 10 to 16-32 bit resolution was preferred.

The additional selection criteria in the case of atmosphere gravity wave (AGW) and transient ionosphere disturbance (TID) investigations were the "major" and "distinguished" missions, targeting in situ measurements of the ionosphere parameters. In details: instrumental and orbital opportunities for simultaneous observations of AGW and TID, i.e. the existence of parallel onboard measurements of neutral and charged particle parameters at ionospheric altitudes $(\leq 350-400 \mathrm{~km}$ or below $800 \mathrm{~km}$ depending on the instrumentation in the moment of or during the measurement). Beside this the sufficient time resolution along the orbit provided by the onboard instrumentation permitting AGW and TID registration over available data sets is also important. A criterion is the existence of prolonged observations (large data sets) with the comparable set of onboard instrumentation for various satellite projects.

By the application of the selection criteria for the satellite listed in Table 1 the following satellite missions were selected for the creation of the IWS: Atmosphere Explorer-C, Atmosphere Explorer-E, Dynamic Explorer 2, GPS system, Variant (Sich1M), DEMETER, and Compass-2. By applying the original (levels 1 and 2) databases of these missions it was possible to produce the level 3 data sets and to create the IWS. After the completion of the basic IWS configuration it is possible to include other satellite databases - archived or provided by operational satellites, e.g. other Atmosphere Explorer data or the new Chibis-M data. 


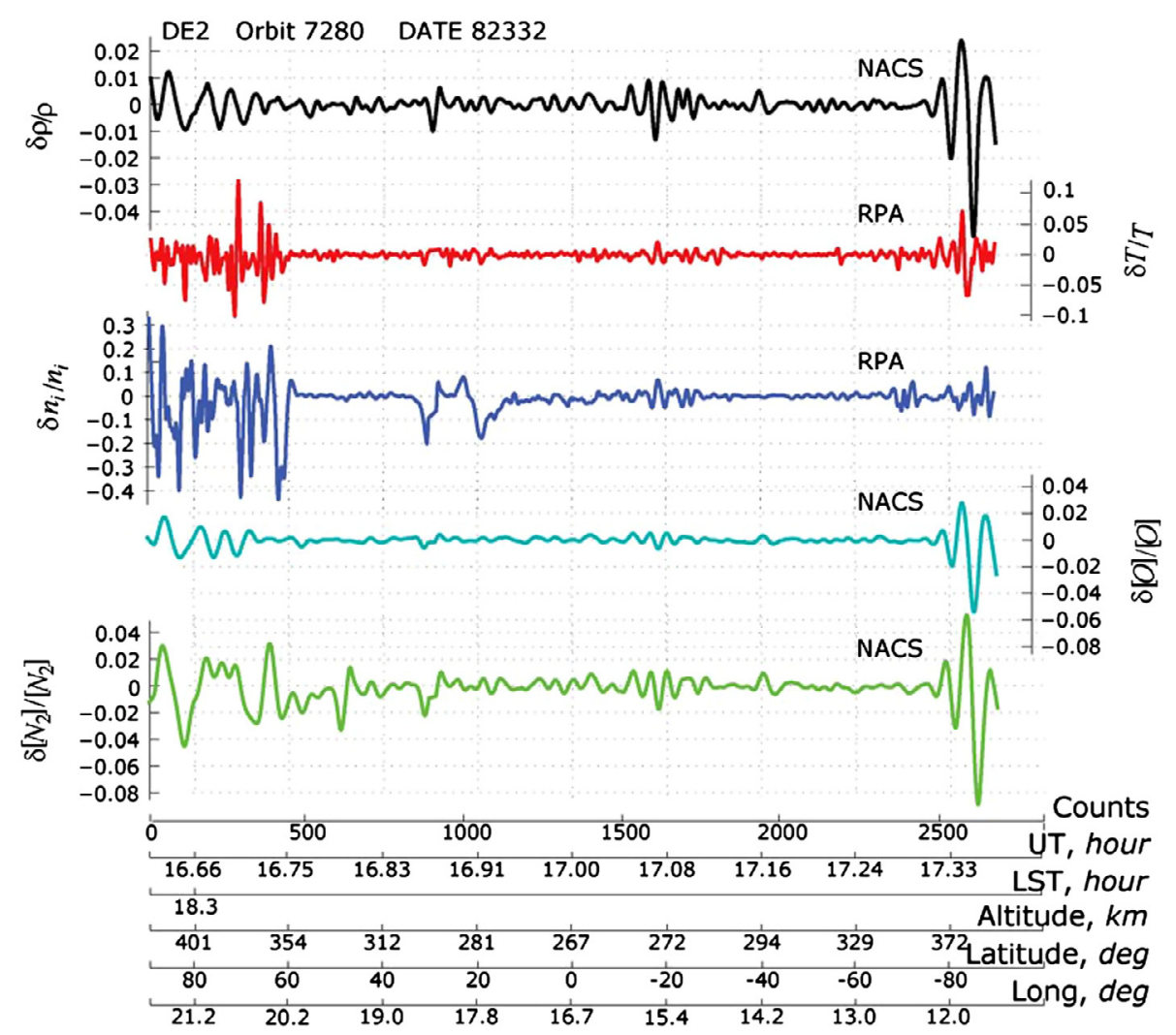

Fig. 9. Plasma and neutral gas variations registered onboard Dynamics Explorer 2 satellite by NACS-instrument (Neutral Atmosphere Composition Spectrometer, detector of the neutral species densities) and RPA-instrument (Retarding Potential Analyzer, detector of the ion density and temperature). Measurement data are filtered in bandwidth $100-1000 \mathrm{~km}$. There are shown (from top to bottom) relative perturbations in neutral density $\delta \rho / \rho$, ion temperature $\delta T / T$, ion concentration $\delta n_{i} / n_{i}$, atomic oxygen $\delta[\mathrm{O}] /[\mathrm{O}]$ and molecular nitrogen $\delta\left[\mathrm{N}_{2}\right] /\left[\mathrm{N}_{2}\right]$ concentrations. Counts are binding to time, altitude, and geographical coordinates of the satellite. AGW waveform is clearly distinguishable in neutral gas parameters. Variations of plasma parameters are noisier being partly caused by AGW, partly by different causes.

\section{Ionosphere transients, wave-like phenomena in the ionosphere}

As it is well known the investigation of the atmosphere-ionosphere transients is an actual problem in the geo- and Space Weather research. However, a catalog-like overview of these phenomena especially of the transients appeared in satellite records is a task often subject of updating with the measurements of the operating ionospheric satellites, today e.g. by the data actually coming from the Chibis-M satellite. But to create an IWS database an actual, state-of-the-art summary of our knowledge about the satellite recorded ionosphere transients was necessary. In the first phase of the planning of IWS we made this summary.

It was possible to define two main classes of the wave-like phenomena in the ionosphere satellite records: electromagnetic wave-like phenomena (EMW) and non-electromagnetic, atmosphere wave-like phenomena. As a consequence of the measuring techniques the number of the types in the EMW class is much higher in this moment as the number of the types of non-electromagnetic, atmospheric transients. It is characteristic that the complete catalog of these phenomena in the case of ionospheric satellites differs from the catalog of ground-based and magnetospheric satellite transient phenomena.

\subsection{Electromagnetic wave-like phenomena (EMW)}

(a) Whistlers, which are ultra-wide-band (UWB) signals generated (mostly) by lightning and propagating through the magnetized plasma (Helliwell 1965) in the magnetosphere and ionosphere nearly along the geomagnetic field lines in ducted or non-ducted modes, or in oblique direction. These signals have right-hand rotating polarization, i.e. these are the electron whistlers. In most cases the whistlers have definite, sharp character in FFT patterns (Fig. 1a), however, in some cases diffuse whistlers also appear. Whistlers are important sounders of the parameters of the traversed ionized medium, of the magnetosphere. Recording whistlers is possible both on board of satellites and at ground stations. Precise UWB theoretical model description of whistlers exists. In Figure 1a the FFT dynamic spectrum of a ducted whistler group is presented which propagated through the magnetosphere in a path about $30,000 \mathrm{~km}$ long between two layer-like inhomogeneities in a waveform with guided mode number 3 , as it was confirmed by a full-wave UWB theoretical model (Ferencz et al. 2001). The result of the modeling is in Figures $1 \mathrm{~b}$ and $1 \mathrm{c}$.

(b) Fractional hop (short path, $0^{+}$) whistlers are UWB signals propagating through the ionosphere from the troposphere to the LEO ionospheric satellite, see in the upper picture of Figure 2.

(c) Ion-whistlers, which have left-hand rotating polarization in whistler-mode propagation (Ferencz et al. 2001; Hughes \& Rice 1997). In the lower picture of Figure 2 it is possible to see proton (higher upper asymptote) 


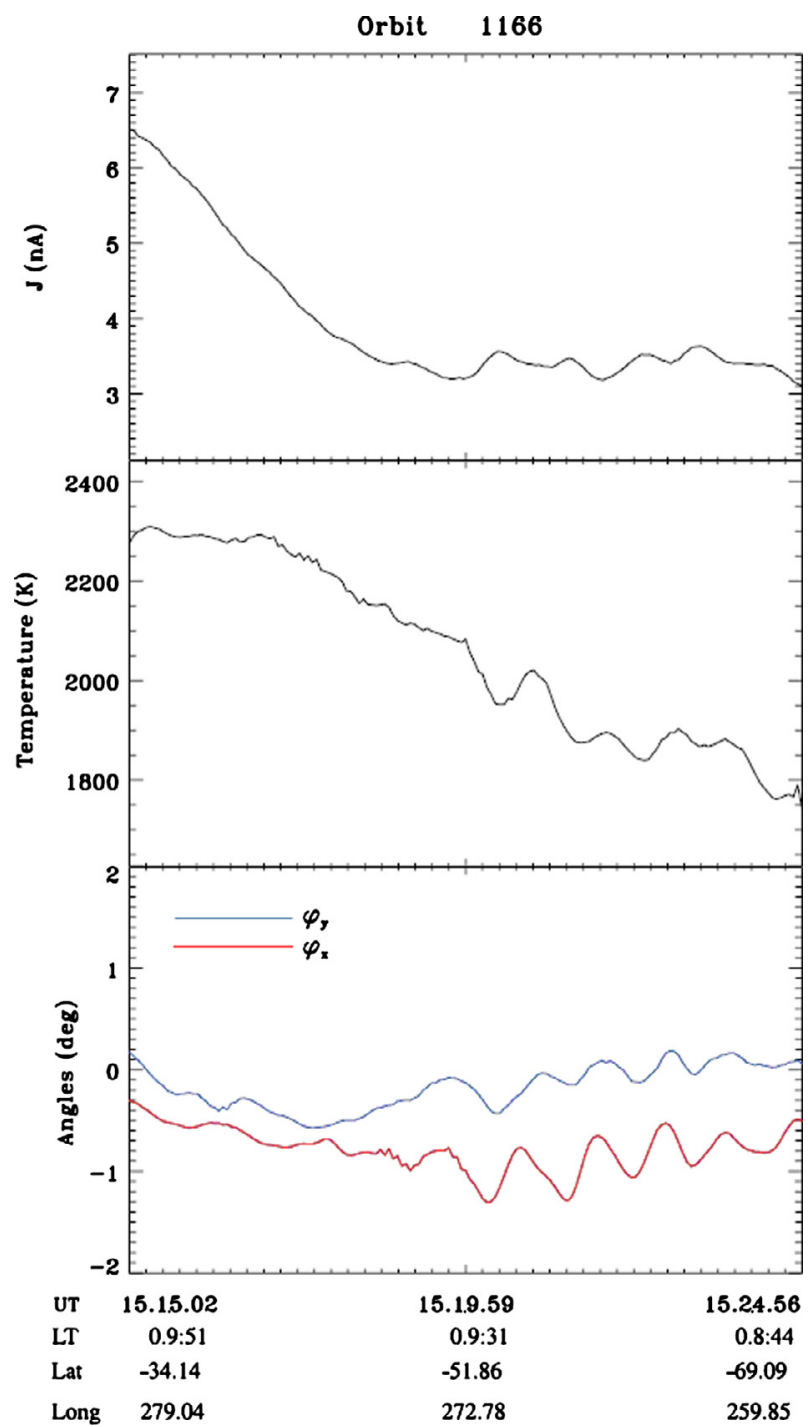

Fig. 10. TID registration by DEMETER IAP instrument at altitude of $730 \mathrm{~km}, 20$ September 2004 . Ion density variations, presented by saturation ion current, show wave-like structure from mid to auroral latitudes. $\mathrm{Ni}$ ion density variations within TID signature anticorrelate with $\mathrm{Ti}$ ion temperature and ion drift (presented by arrival angles from DM sensor $\varphi_{x}, \varphi_{y}$ ).

and helium (lower upper asymptote) whistlers accompanied to the right-hand propagating short path fractional hop electron whistler.

(d) Low-latitude whistler pairs (trapezoid whistler signals): On board of ionospheric LEO satellites often appear short path fractional hope whistler pairs with common lightning origin at lower magnetic latitudes. This type of signal contains two components with slightly different dispersions. A typical series of low-latitude whistler pairs is in Figure 3.

(e) Spiky whistlers $(\mathrm{SpW})$, which UWB signals are generated by lightning and propagated in the Earth-ionosphere wave-guide before the out-coupling from this waveguide to traverse the ionosphere and going into the magnetosphere (Ferencz et al. 2007).

(f) Swallow-tailed whistlers (STW): These UWB signals have whistler character in their main trace, also exhibiting additional wave structure as strong signal trace splitting and deviation on broadband spectra, with a shape of swallow-tail (Ferencz et al. 2010; see in Fig. 5). In this moment no accepted or proposed explanation of the generation, of the origin of these signals exists.

(g) Crossed trace or X-shaped whistlers, which UWB signals form signal branch with crossing pattern in an FFT picture and have whistler character; see e.g. in Figure 6a. In this moment no accepted explanation exists. The proposed explanation is: Ducted whistler-mode UWB signals, propagating in different ducted mode wave-form, crossed each other in FFT pattern; see in Figure $6 \mathrm{~b}$. In other cases a regular fractional hop whistler was recorded together with a higher $\mathrm{SpW}$ signal mode propagating alone; see in Figure $7 \mathrm{a}$ and the modeled FFT in Figure $7 b$ (Ferencz et al. 2007).

(h) It is still an important, open question in the lower atmosphere-ionosphere coupling investigations, which kind of electromagnetic transients appeared simultaneously in ground-based and satellite measurements (see e.g. in Chum et al. 2006; Inan et al. 2007; Parrot et al. 2008, 2009; Błęcki et al. 2009; Lefeuvre et al. 2009; Nemec et al. 2010).

(i) Diffuse signals: chorus, hiss, triggered emissions, etc.: These signals have no such characteristic shapes as the whistler-like signals. The investigation of these signals is important, however, the investigation methods are only in the research-development phase (Nunn et al. 2003; Meredith et al. 2006; Golden et al. 2011).

(j) Unclassified, anomalistic waveforms, i.e. strange signals: These phenomena are coherent signal structure appearing in the records of ionospheric satellites and in this moment no valid physical model or idea exists about the origin of these signals (Ferencz et al. 2010). See an example in Figure 8.

\subsection{Non-electromagnetic, atmosphere transients, wave-like phenomena}

(a) Atmosphere gravity wave $(A G W)$ is one of the main types of neutral upper atmosphere oscillations with periods from approximately $10 \mathrm{~min}$ up to $1-2 \mathrm{~h}$ and horizontal wavelengths from a few hundred $\mathrm{km}$ up to a half-two thousand km. AGW appears as result of arbitrary atmosphere deviation from the hydrostatical equilibrium (caused by the air heating, ion dragging, Kelvin-Helmholtz instability, etc.). The mechanism of AGW propagation consists in cooperative action of two restoring forces upon the air parcel: buoyancy force and pressure disturbance. Classic papers (Hines 1960, 1974; Francis 1975; Kato 1980; Mayer et al. 1990; Hocke \& Schlegel 1996) give detailed description of the AGW at ionospheric heights.

In terms of the theoretical hydrodynamics, there are two basic environmental parameters that give complete information about AGW; these parameters are density and temperature variations. In parallel, AGW induces in the upper atmosphere the forced oscillations of individual gas component concentrations ( $\delta[\mathrm{O}], \delta\left[\mathrm{N}_{2}\right]$, etc.), the oscillations of ion concentration and velocity (latter is treated as traveling ionosphere disturbance, TID), and the Earth magnetic field pulsations. Figure 9 shows a pattern of AGW detected in situ onboard of Dynamics Explorer 2 satellite.

(b) Traveling ionospheric disturbance (TID) is ionospheric plasma density oscillation caused by AGW upward 
Table 2. Summary of the known wave recognition methods.

\begin{tabular}{|c|c|c|c|c|}
\hline Name of the procedure & Target phenomenon & $\begin{array}{l}\text { Key element of the } \\
\text { procedure }\end{array}$ & $\begin{array}{l}\text { Related phenomena, } \\
\text { if known }\end{array}$ & $\begin{array}{l}\text { Possible application in } \\
\text { IWS creation }\end{array}$ \\
\hline $\begin{array}{l}\text { Automatic Whistler Detection and } \\
\text { Analyzer algorithm, AWDA }\end{array}$ & $\begin{array}{l}\text { Whistlers, in } \\
\text { ground-based } \\
\text { measurements }\end{array}$ & $\begin{array}{l}\text { 2D image } \\
\text { correlation in } \\
\text { dynamic wave } \\
\text { spectra }\end{array}$ & $\begin{array}{l}\text { Energetic particles, } \\
\text { hiss; Space Weather, } \\
\text { earthquake, etc. }\end{array}$ & $\begin{array}{l}\text { After adaptation to } \\
\text { satellite recorded data }\end{array}$ \\
\hline $\begin{array}{l}\text { Automated Chorus and Hiss detection } \\
\text { method }\end{array}$ & $\begin{array}{l}\text { VLF chorus, hiss, } \\
\text { in ground-based } \\
\text { measurements }\end{array}$ & $\begin{array}{l}\text { Artificial neural } \\
\text { network in spectra }\end{array}$ & $\begin{array}{l}\text { Magnetic records, } \\
\text { energetic particles; } \\
\text { Space Weather, etc. }\end{array}$ & $\begin{array}{l}\text { Only few information } \\
\text { is known from the } \\
\text { method }\end{array}$ \\
\hline $\begin{array}{l}\text { Bandpass filtering of TEC time series } \\
\text { computed from bi-frequency GPS } \\
\text { receiver's RINEX data }\end{array}$ & TID & $\begin{array}{l}\text { Time domain } \\
\text { digital filtering }\end{array}$ & $\begin{array}{l}\text { Space Weather, } \\
\text { earthquake, etc. }\end{array}$ & Without modifications \\
\hline $\begin{array}{l}\text { Bandpass filtering in a narrow range and } \\
\text { wave-type verification }\end{array}$ & AGW and TID & $\begin{array}{l}\text { Time domain } \\
\text { digital filtering }\end{array}$ & $\begin{array}{l}\text { Space Weather, } \\
\text { earthquake, etc. }\end{array}$ & $\begin{array}{l}\text { Used along with newly } \\
\text { elaborated processing } \\
\text { techniques }\end{array}$ \\
\hline $\begin{array}{l}\text { General algorithm for transient signal } \\
\text { detection in time domain data series }\end{array}$ & $\begin{array}{l}\text { Deterministic } \\
\text { transients }\end{array}$ & $\begin{array}{l}\text { Adaptive pattern } \\
\text { recognition in time } \\
\text { domain }\end{array}$ & Not specified & $\begin{array}{l}\text { Only to solve special } \\
\text { tasks and with further } \\
\text { development }\end{array}$ \\
\hline
\end{tabular}

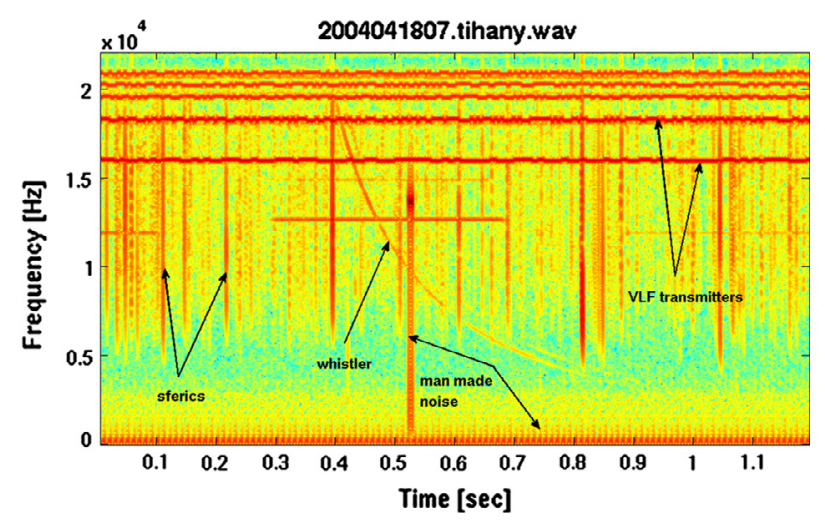

Fig. 11. A typical ground-based VLF (whistler) record in dynamic spectrum (relative wave intensities in logarithmic color scale). This data set was recorded at a mid-latitude ground-based station (Tihany, Hungary, $L=1.8$ ) on 18.04.2004 07.00 UT.

penetration throughout thermosphere and ionosphere. TID can affect topside F-region altitudes. Above the collision atmosphere boundary (exobase) TID structures propagate along the magnetic field lines. In general, into the lower F-region, TID has the same temporal and spatial scales that AGW. In parallel, plasma variations at orbital heights may be caused by a number of other events, either influenced from below or due to the magnetosphere-ionosphere coupling processes. Therefore, the main characteristics of wave-like plasma density structures observed at satellite orbit strongly depend on geographic position and local solar time at the observational area, geomagnetic activity level, etc.

TIDs propagate in global scales from auroral ovals toward low latitudes (Balthazor \& Mofett 1997) and/or across polar caps (Cai et al. 2011) appearing as oscillations in F-region plasma parameters, like plasma density, temperature, drift velocity, mean ion mass, etc. These oscillations provide significant fluctuations in the plasma parameters registered by in situ

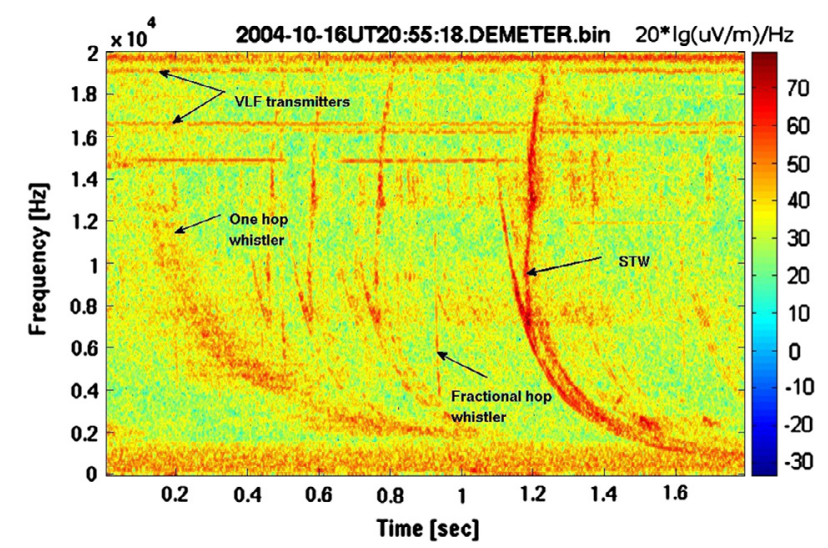

Fig. 12. A typical satellite-based VLF dynamic spectrum. The raw data were recorded on board of DEMETER satellite in 16.10. 2004 UT 20.55.18.

observations at low Earth orbiting satellites and/or remotely by various ground-based radio-sounding techniques; between these possibilities one of the most effective technique is the measuring of the total electron content (TEC) along the GPS signal propagating path crossing the whole ionosphere (Crespon 2007). Depending on the spatial scale TIDs are commonly divided into middle-scale-TIDs (MS-TIDs) with horizontal wavelengths about several hundred $\mathrm{km}$ and large-scale-TIDs (LS-TIDs) with horizontal wavelengths more than thousand $\mathrm{km}$. An example of MS-TID, as observed on 20 September 2004 (orb. 1166) at $\sim 700 \mathrm{~km}$ altitude by IAP instrument onboard DEMETER satellite, is shown in Figure 10 (Berthelier et al. 2006).

\section{Wave recognition and extraction methods}

It is clear that after the levels 1 and 2 data processing of the (ionosphere) satellite raw data flow the transient, wave-like phenomena listed in Section 4 are not selected from the data flow, i.e. these phenomena are there somewhere in the measured data mass. However, before starting of the research work of a given 
C. Ferencz et al.: IWS: Ionosphere Waves Service

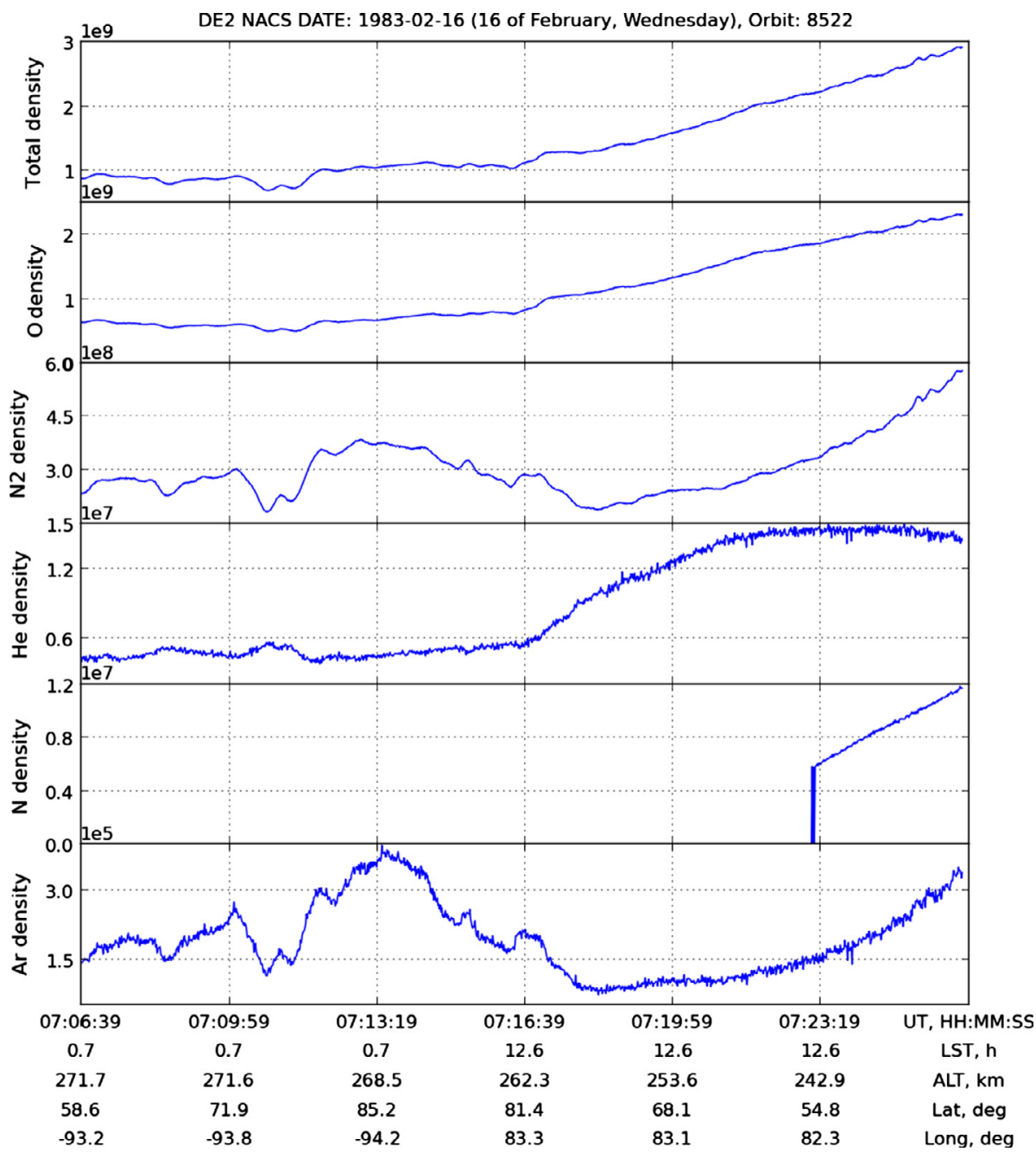

Fig. 13. Distribution of neutral atmosphere components concentration along DE 2 orbit.

task (Space Weather research, earthquake investigations, research of the generation or propagation processes of these phenomena in details, etc.) it is necessary to find these wavelike phenomena in the whole data mass. Therefore it is essentially important to produce the level 3 processed data base, the IWS. However, to find the wave-like phenomena in the total data flow is possible only with automatic or at least semi-automatic methods. This means the application of intelligent data processing methods and therefore a minimum requirement is the application of the up-to-date computer techniques. Earlier the development and application of these methods was limited by the non-adequate level of the computer techniques. As a consequence of this situation the transient-searching techniques are really very new and we have no such tool for the whole set of the important and interesting transients. It is necessary to overview of the existing methods and after the necessary adaptation and/or development of these methods it is possible to create the IWS.

\subsection{Short overview of the known wave-phenomena recognition methods}

As we know, only a few methods exist. We found only five automatic or semi-automatic methods, which could be used to extract the data of the wave-like phenomena from the raw or first or second level data mass of a satellite measurement, see in Table 2.

\subsubsection{Automatic Whistler Detection and Analyzer (AWDA) algorithm}

The AWDA algorithm was developed by the Eötvös University Space Research Group for ground-based recorded VLF electromagnetic data. The procedure detects and optionally analyzes real time in VLF recordings the lightning generated whistlers, propagating in terrestrial plasma environment (Lichtenberger et al. 2008). Automatic whistler detection is based on 2D correlation technique, carried out in dynamic spectra of high resolution ELF/VLF recordings. After proper removal of "noises" in whistler point of view, like spherics, signals of strong narrow-band VLF transmitters, band limited data is used for data processing. The whistler selection in the data flow is based on thresholding of output of synthetic (theoretical UWB) whistler and recorded data correlation, where the fundamental algorithm parameters are finally adjusted by site specific calibration procedure. The detection speed is within real time, selected whistlers are stored separately in short recording junks. Automatic detection of whistlers provides reliable occurrence statistics, with orders of magnitude larger number of cases than in traditional data processing methods using human 


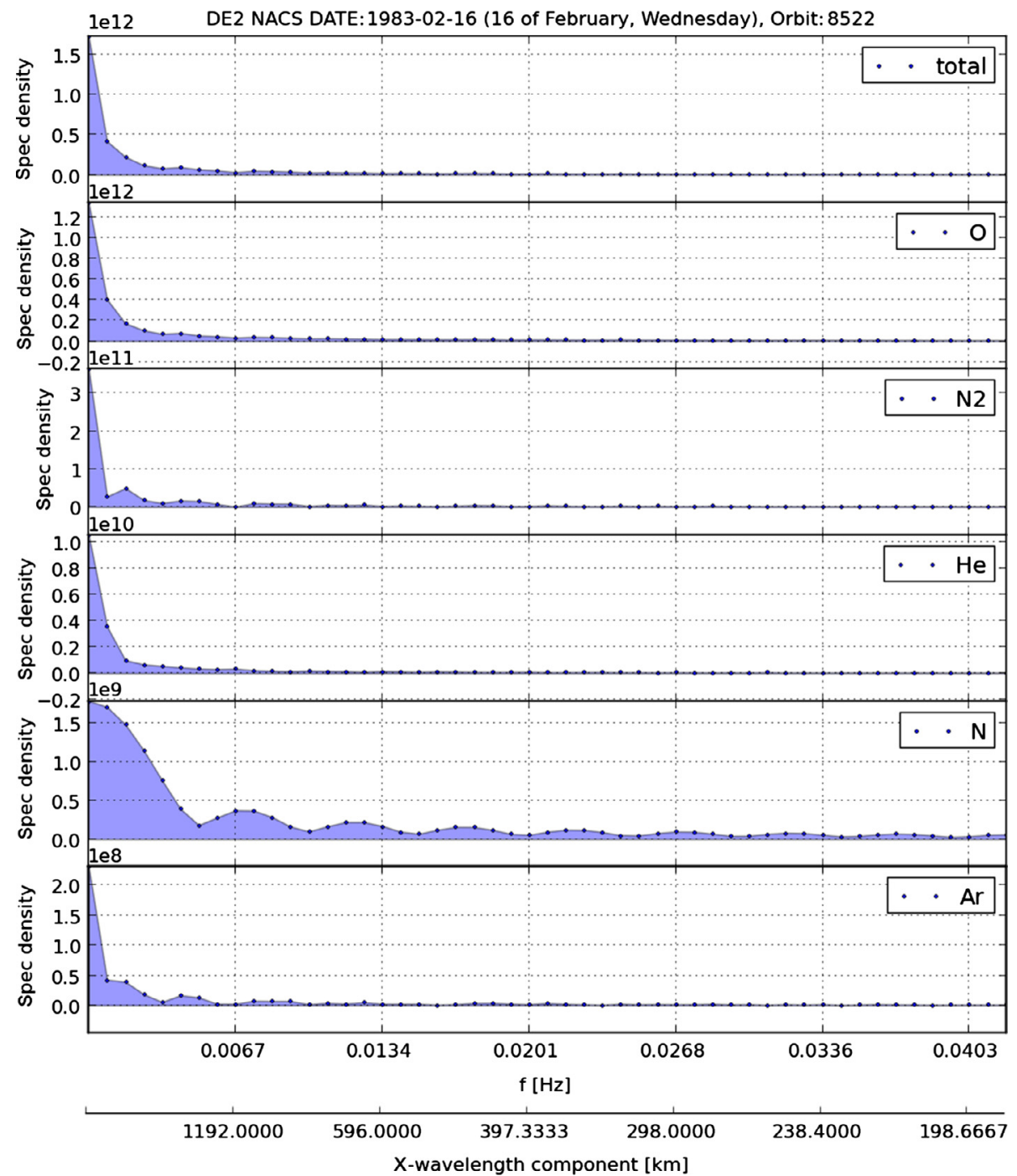

Fig. 14. Spectral composition of neutral atmosphere components concentrations, same as illustrated in Figure 13. Spectral power is displayed as function of horizontal wavelengths.

intervention. Detected whistlers yield input wave set of automated whistler analysis procedure. Whistler analysis aims to gather medium parameters from whistler signal characteristics. Based on a virtual whistler trace inversion procedure, best fit parameters of selected plasmasphere definitive model is extracted. The AWD system is in successful, real time operation in the global AWDANet network and the AWDA real time version is under installation in this network too (Lichtenberger et al. 2010; Collier et al. 2011).

\subsubsection{Automated chorus and hiss detection algorithm}

The procedure was developed by the Stanford University Electrical Engineering Department for processing of ground-based VLF data, recorded during 10 years on Palmer Station, Antarctica and to identify and differentiate chorus and hiss emissions in ELF/VLF recordings (Golden et al. 2011). The algorithm consists of three major processing steps. First, sferics and power line hum are removed from the broadband data. Second, individual events are detected and a set of 19 scalar event parameters are determined. Finally, on the basis of the parameters, detected events are categorized by a sequential pair of neural networks as chorus, hiss, or noise. Chorus and successive hiss emissions are proven to correlate with geomagnetic activity. Comparison of chorus/hiss statistics with Solar/geomagnetic indices is an obvious subject of investigation. The time base accuracy is good, however, it is exactly not known. The practical details of the method were not reachable during the construction of the IWS.

\subsubsection{Bandpass filtering of TEC time series computed from bi-frequency GPS receiver's RINEX data}

TEC time series are extracted from GPS phase measurements for each receiver-satellite link. The geometry free combination is computed to provide relative value of TEC, a bandpass filtering is applied (Lognonné et al. 2006; Crespon et al. 2007) and the data forms a web-service produced by Noveltis. So we obtain filtered TEC time series in which Large Scale TIDs (MSTID) and Medium Scales TIDs (LSTID), depending to the filtering band, can be observed. The wave recognition was usually done by human expertise (Afraimovich et al. 2000, 2002, 2009; Calais \& Haase 2003; Artru et al. 2005; Hayashi et al. 2010; Makela et al. 2011). 

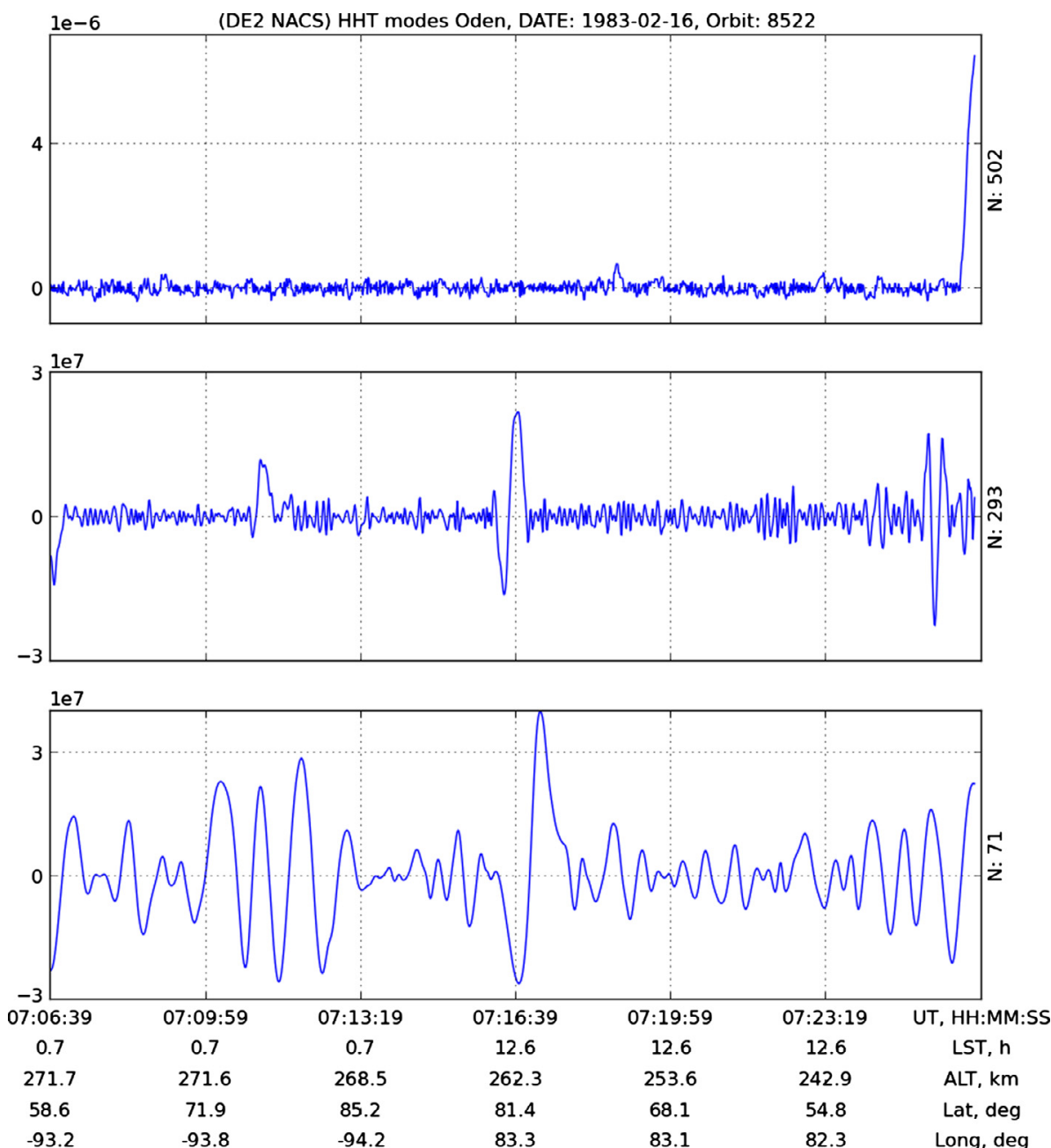

Fig. 15. Hilbert-Huang empirical modes after sifting: instrumental high frequency noises (upper panel), middle-scale AGW (middle panel), and large-scale AGW (lower panel).

\subsubsection{Bandpass filtering in a narrow range and wave-type verification}

The method was adapted for AGW extraction from a satellite data set in Space Research Institute NASU-NSAU. Method is based on extensive practical experience, and there are convincing examples of its efficacy (Fedorenko et al. 2005; Skorokhod \& Lizunov 2012), but to that moment there is no theoretical and methodological substantiation necessary to fully automate the process.

\subsubsection{General algorithm for transient signal detection in time domain data series}

The method was developed for and successfully applied in ground-based narrow-band VLF recordings (Steinbach 2006). Time domain data sequences often exhibit transients in various characteristic time ranges. These variations may cover large dynamics in amplitudes, usually also overlap in time. Without known physical source mechanism they are considered as "noise". One typical shape is e.g. quick change and slower relaxation in recorded signal amplitude and/or phase data. With appropriate training process this algorithm is also possible to apply to other deterministic type of transients, appearing in time sequences recorded on board of satellites.

\subsection{Wave recognition methods actually applied in the creation of IWS}

Seeing the development goals, the application results and the actual state of the automatic or semi-automatic wave identification methods it was possible to select the methods which are applicable for satellite recorded data sets directly or after a goal-oriented development. See the 5th column of Table 2. Only three methods remain after this selection because the two years long running time of the POPDAT FP7 project for creation of the IWS. However, it is clear that applying these methods the creation of a unique and practicable, goal-oriented IWS database is possible. Further expansion of this IWS is feasible with new wave phenomena and new satellite databases, e.g. with the CHIBIS-M database. The causes of selection were first the fact that in the case of the Automated Chorus and Hiss detection method only few information is known from the method, and in the case of the General algorithm for transient signal detection in time domain data series is applicable in satellite data records only to solve special tasks and with further and 


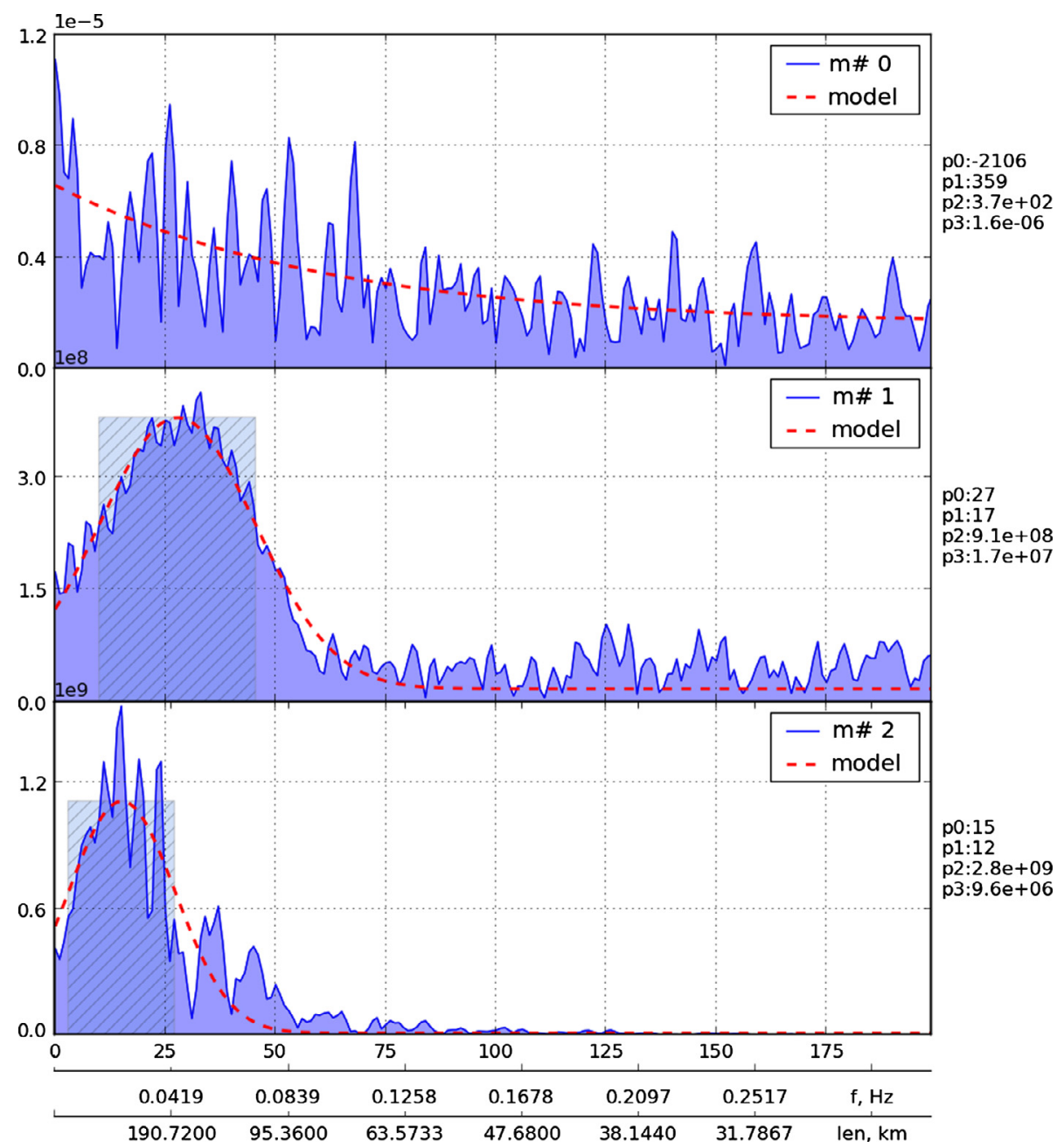

Fig. 16. Spectra of the selected modes. Shaded areas in the middle and the bottom panel indicate effective spectrum width of the wave process.
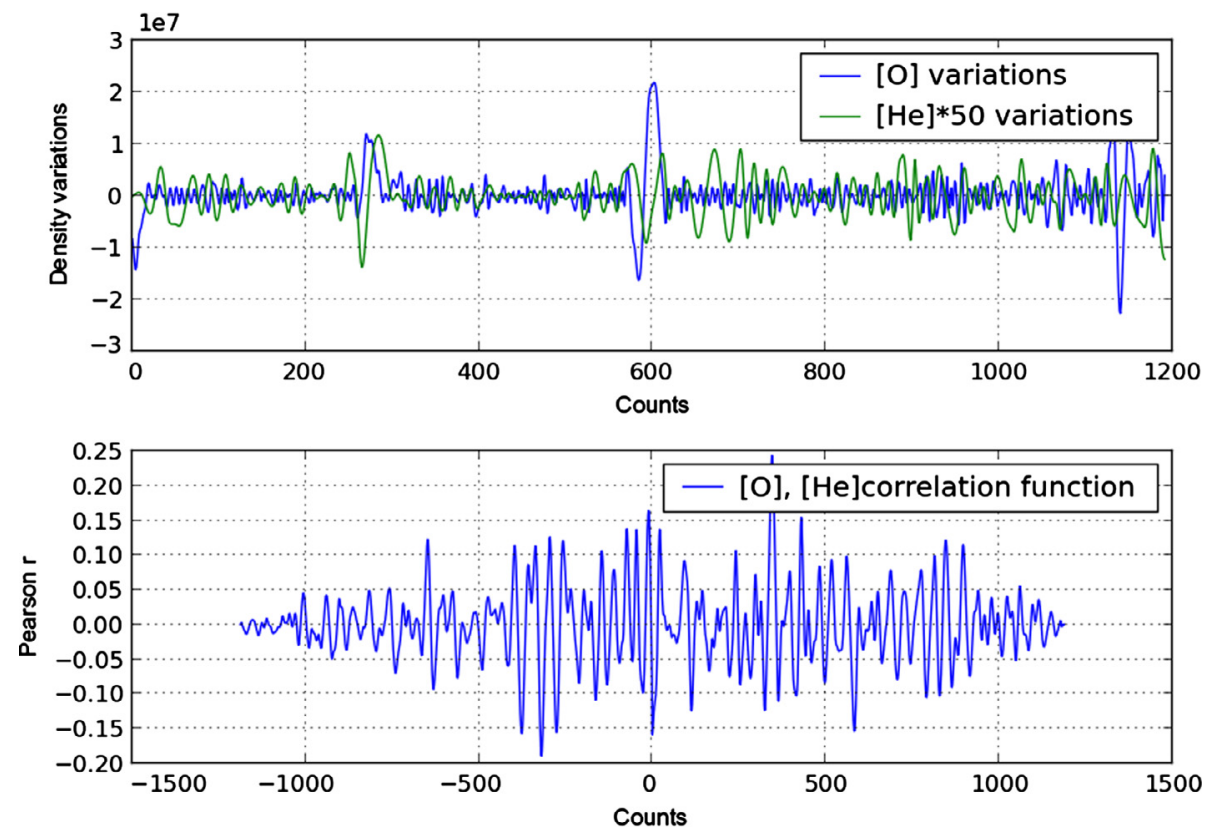

Fig. 17. Wave modes in $[\mathrm{O}]$ and $[\mathrm{He}]$, and their correlation function. 


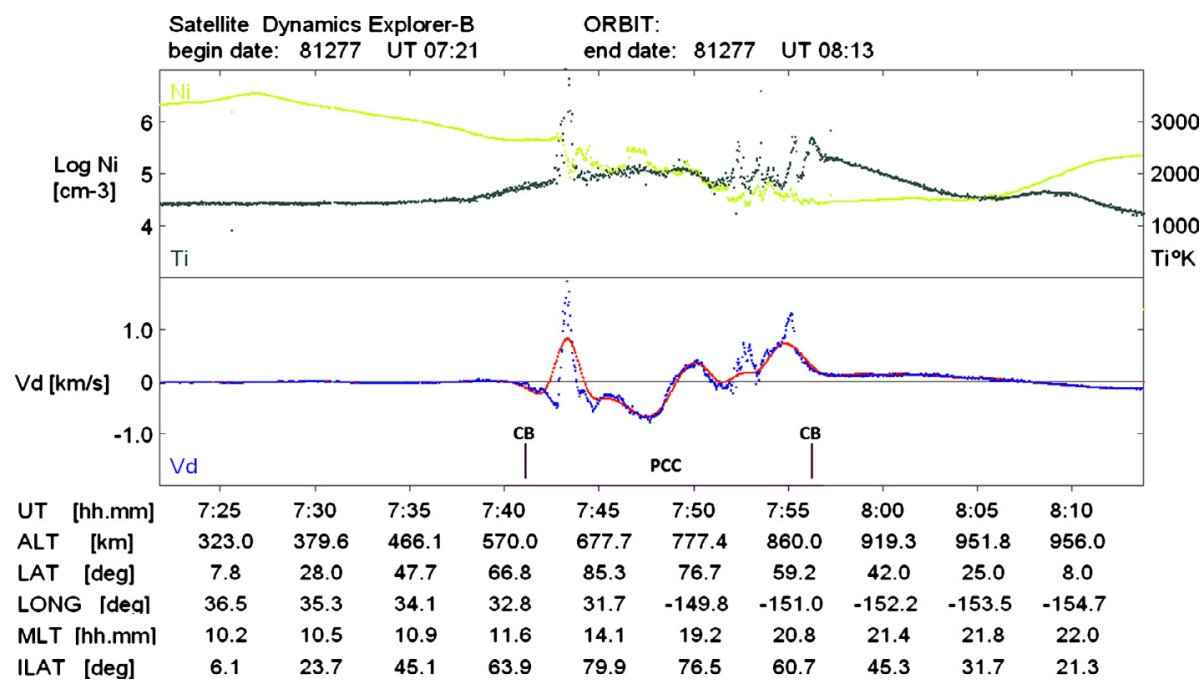

Fig. 18. Ion density, ion temperature (top panel), and transverse horizontal component of ion drift (with spline fitted curve) as measured by RPA/ IDM instrument onboard Dynamics Explorer 2 satellite, see text below for details.

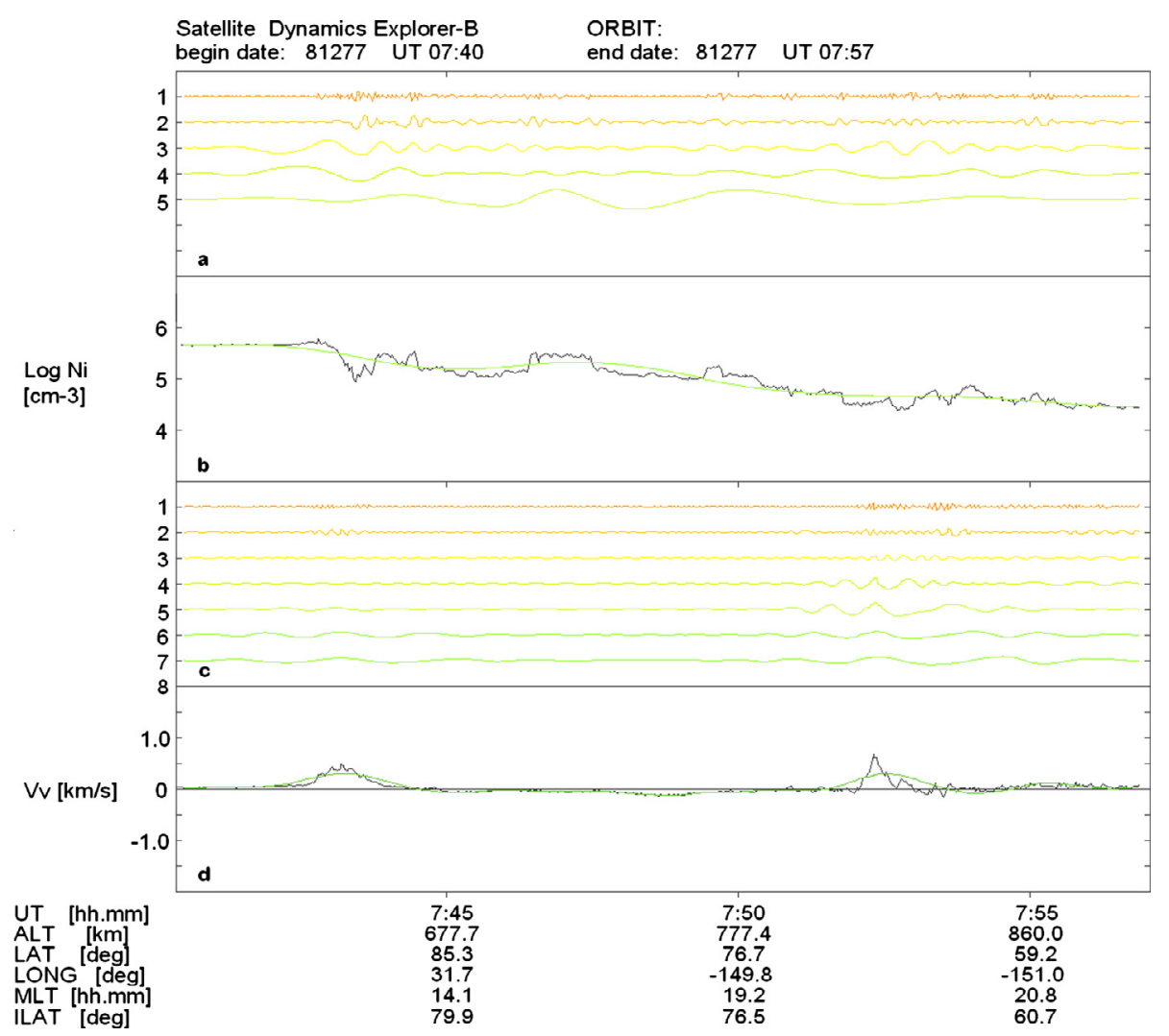

Fig. 19. HHT processed data (absolute values in the same scale) inside PCC zone - see in Figure 14 for Ni and vertical ion drift velocity $V v$ (see text for details). Green line on panels (b), (d) represents last HHT decomposition for given parameter; Ni on (b) and $V v$ on (d) panel.

larger development. In the following we present a short summary of the three methods used in the creation of the IWS.

\subsubsection{Automatic transient electromagnetic, whistler-like phenomena detection in satellite recorded data}

As it is known from earlier works (Lichtenberger et al. 2008) the automatic detection of pre-defined UWB (transient and whistler-like signals) signals in a record containing other strong signals, noise, is a complex task, however, it is solved in the case of ground-based records and this software system is running effectively in the AWDANet system around the globe. However, the structure of the satellite records differ from the ground-based ones. The summary of the original AWD software for ground-based applications is given above in Section 5.1.1 and the details of this method can be found in the cited (Lichtenberger et al. 2008) paper. The main differences between the ground-based and (ionospheric) satellite VLF records are demonstrated in Figures 11 and 12. 
J. Space Weather Space Clim. 4 (2014) A17

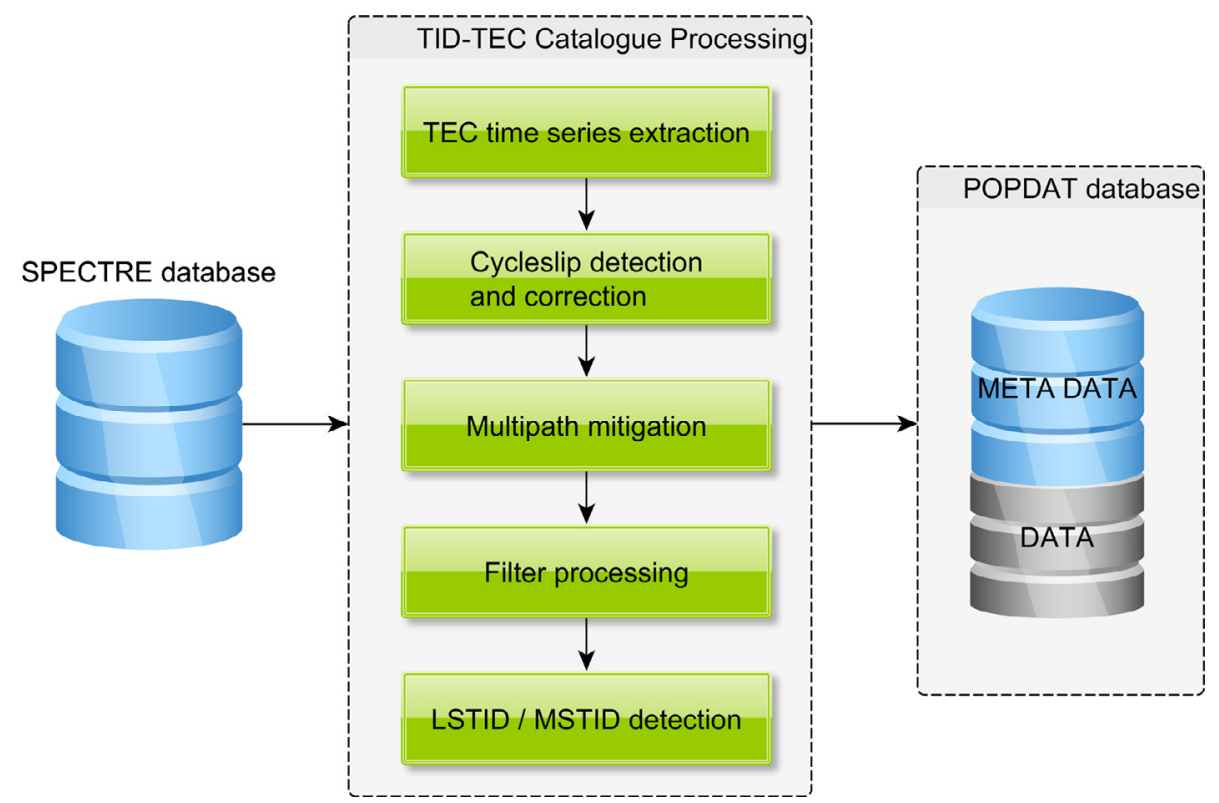

Fig. 20. Logic scheme of the processing chain dedicated to the detection of TID from GPS network.

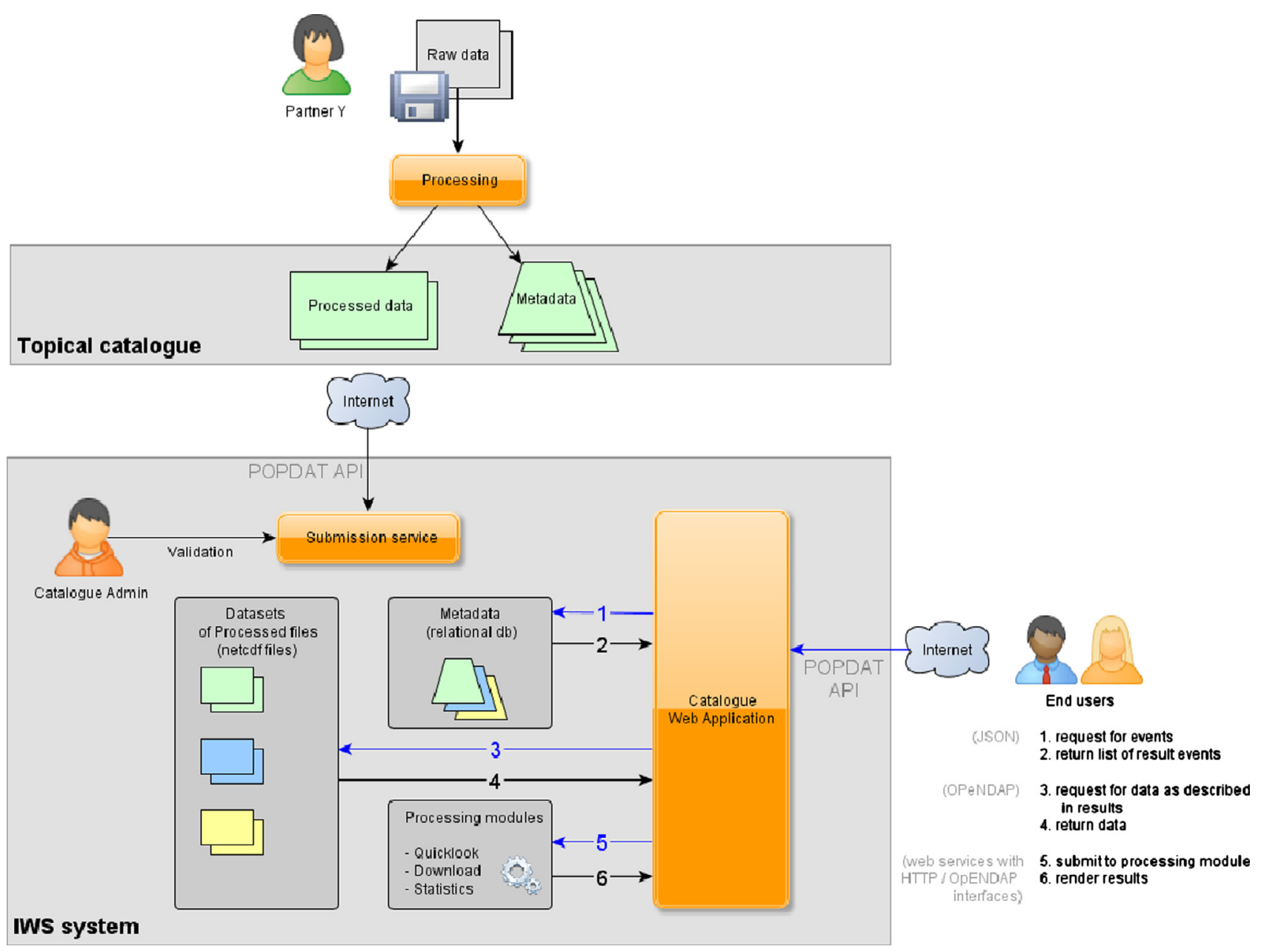

Fig. 21. The functional diagram of the Ionosphere Waves Service. 


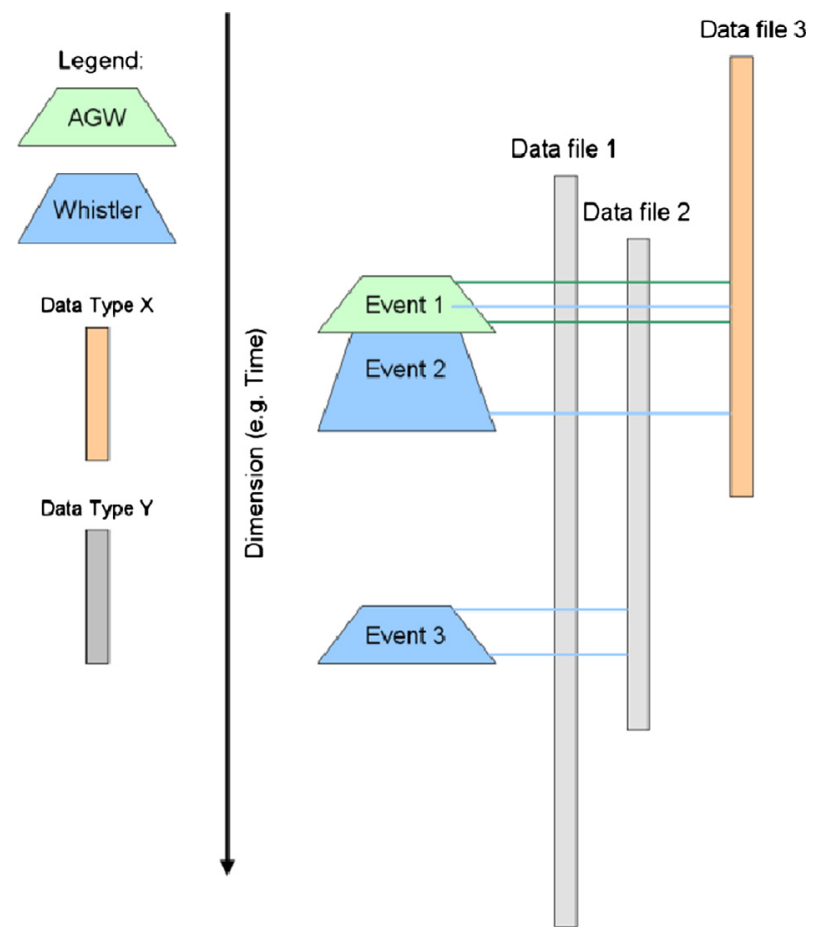

Fig. 22. Ionopshere Waves Service data model overview. Metadata are identified as several type of events (green and blue boxes). Processed data are identified as several type of data (gray and orange bars).

In the case of satellite recorded signals this method is applicable only after an adaptation to the differences of the contents of the ground-based and satellite records. The important differences are the following:

- The propagation paths and circumstances are or could be very different for the ground recorded and satellite recorded whistlers and UWB signals. In the original AWD version the automatically detected signals propagating from one hemisphere of the Earth to the other (see Fig. 11). In the case of the signals recorded on board of satellites the known or supposed propagation paths could be similar or much shorter or totally different than in the case of ground recorded signals (see Fig. 12). Therefore the shape of the whistler-like signals could be similar or totally different than the shape of the ground recorded whistlershape.

- VLF transmitter signals (strong horizontal lines above $12 \mathrm{kHz}$ ) are present in the satellite records too, and the elimination of these signals is necessary in our investigations.

- Sferics and tweeks are not present in satellite records, however, relatively strong and nearly vertical-like signals, the fractional hop whistlers are in these records and these signals are important for research, i.e. these are not "noises" in satellite records.

- The power line harmonics are not too strong or are not present, but the spacecraft (satellite, ISS, etc.) can produce EMC problems and noise-like turbulences, and the elimination of these signals is necessary.

Therefore the noise-filtering part of the AWD must change in satellite applications and the signal-shape description, the theoretical form if the interesting signals must contain the signal shapes propagating in different paths to the satellite. The elimination of the onboard EMC noises is necessary.

For application of the AWD method it is necessary to have a correct theoretical (mathematical) description of the searched UWB signal shape-class. In the case of more electromagnetic wave-like phenomena this description is not known, it is an actual task in fundamental research and therefore it was necessary to exclude such kind of UWB phenomena from the creation of the IWS. The classes which were omitted from the POPDAT project and from the creation of the IWS are:

- Hiss and chorus detection (the method is in experimental phase).

- Triggered emissions (no idea is known for the automatic detection).

- Strange signals (no definition of these signals is known).

The investigation of these signals is possible in the traditional way by researchers.

By applying an advanced AWDSat software system it is possible the automatic detection and selection of the whistlers and whistler-like phenomena and the incorporation of these data and the derived metadata into the IWS:

- The AWDSat-WHF for the automatic detection of the normal (typical single hop) whistlers in the satellite recordings. This algorithm can detect and select automatically the whistlers in the data sets recorded on board of satellites, if these whistlers have a higher dispersion, i.e.

$$
D>35 \sqrt{s} \text {. }
$$

The typical dispersion range of the normal whistlers in satellite records is:

$$
D=45 \sim 200 / 300 \sqrt{s} .
$$

- The AWDSat-LIWHF for the automatic detection of the longer path fractional hop whistlers in the satellite records. This algorithm can detect and select automatically the whistlers in the data sets recorded on board of satellites, if these whistlers have smaller dispersion, i.e.

$$
D=8 \sim 35 \sqrt{s}
$$

- The AWDSat-SIWHF for the automatic detection of the short path fractional hop whistlers in the satellite records. This algorithm can detect and select automatically the whistlers in the data sets recorded on board of satellites, if these whistlers have small dispersion, i.e.

$$
D \leq 8 \sqrt{s} .
$$

The typical dispersion values of these whistlers in satellite records are:

$$
D=0.8 \sim 8 \sqrt{s}
$$

- The AWDSat-WHPF for the automatic detection of SIWHF and LIWHF signals if they appear simultaneously, the SIWHF signal appears before the LIWHF signal and the time gap between the two signals is in the order of $100 \mathrm{~ms}$. 


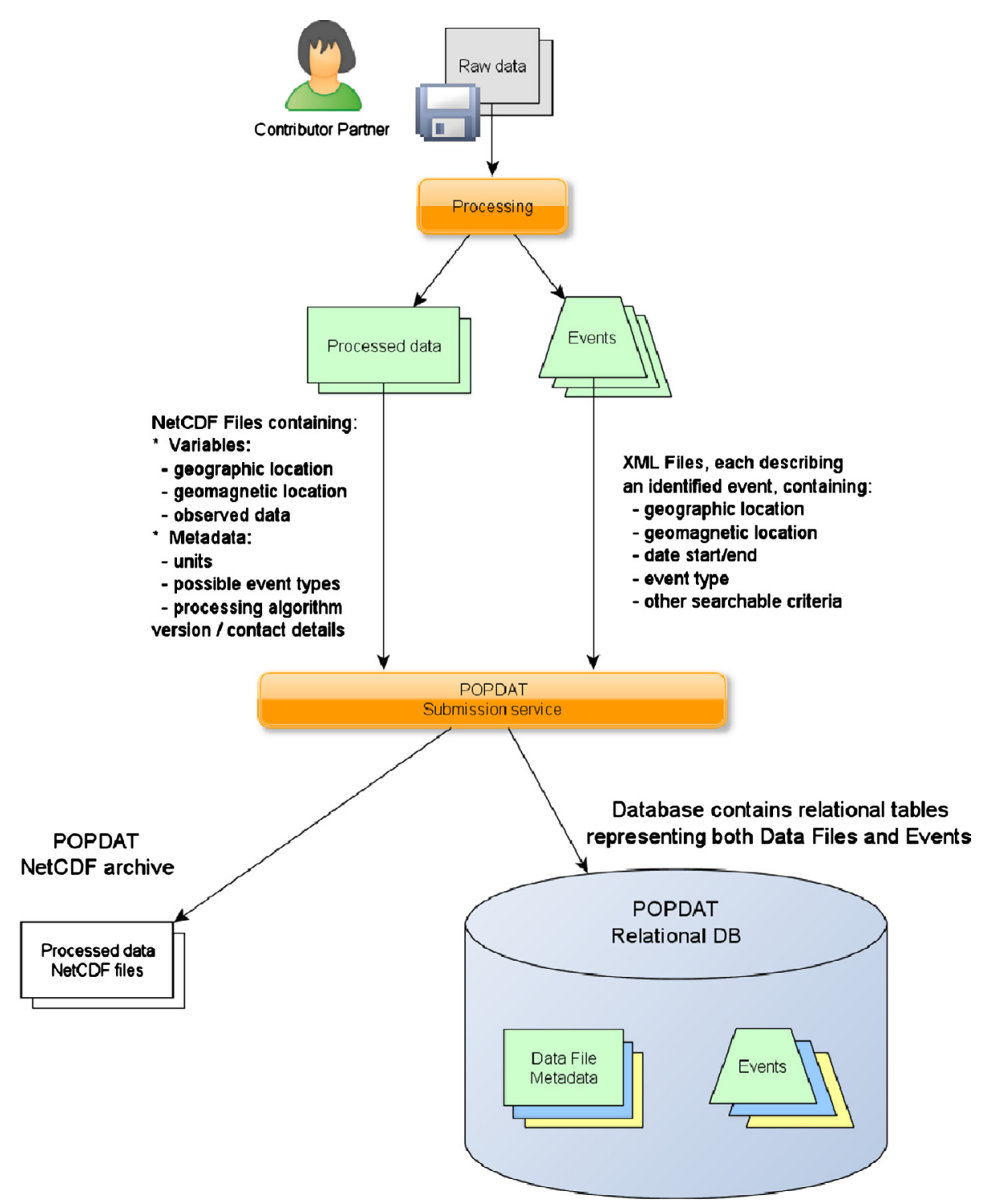

Fig. 23. Metadata (events) and Data (processed data) submission flow in the Ionosphere Waves Service system.

The AWDSat solved the problem of the automatic detection of the classes mentioned above and the results are metadata with the occurrence of the events in the listed four classes and samples of detailed wave-forms of detected events. Since tthe AWD concept is based on adaptive filtering, the procedure is highly sensible for "noise" patterns, and that is handled in the first phase. This procedure section had to be changed according to the difference of onboard and ground-based recording characteristics. Most remarkably in the beginning phase the original AWD omits the "vertical" spectral lines in dynamic spectra which are great amplitude sferics and not whistlers, therefore these lines in the ground-based measurements are the dominant noises to get rid of. However, in the satellite records the SIWHF phenomena show a columnar pattern in VLF spectra above $5-10 \mathrm{kHz}$. These signals are the principal electromagnetic phenomena measured in situ in the terrestrial plasma environment, related to thunderstorm activity research. (Note that local, manmade EMC-noises may also appear in satellite recordings, elimination of these signal parts, overlapping short whistler impulses require careful analysis.) Satellite recordings exhibit typically much larger number of whistler occurrences than recordings on the ground. This affects the minimum temporal separation between independently detected events, the detection efficiency. New detector settings had to be found and set so that frequent sequences of short path whistlers to make it possible to detect them as separate events. Further, whistlers detected in space-born datasets have dispersion values falling in a rather wide range, due to the diversity of the possible propagation paths. These waves may appear overlapped according to the variability of their possible signal dispersions. As a completely new concept, applied and tested first in satellite recordings within the POPDAT IWS database preparation, after one above-mentioned detection turn on a given type, values representing the detected events are nullified from the spectra, lowering the noise level and allowing a better detection in its next loop turns. This is best performed if the most dominant type, determined also automatically is processed first. This kind of data processing is running in the creation of the IWS electromagnetic wave-like phenomena goal-oriented database.

The investigation of other electromagnetic phenomena is also possible as "sub-classes" of the four classes listed above by the direct investigation of the automatically detected whistler-like events by researchers. These are: 


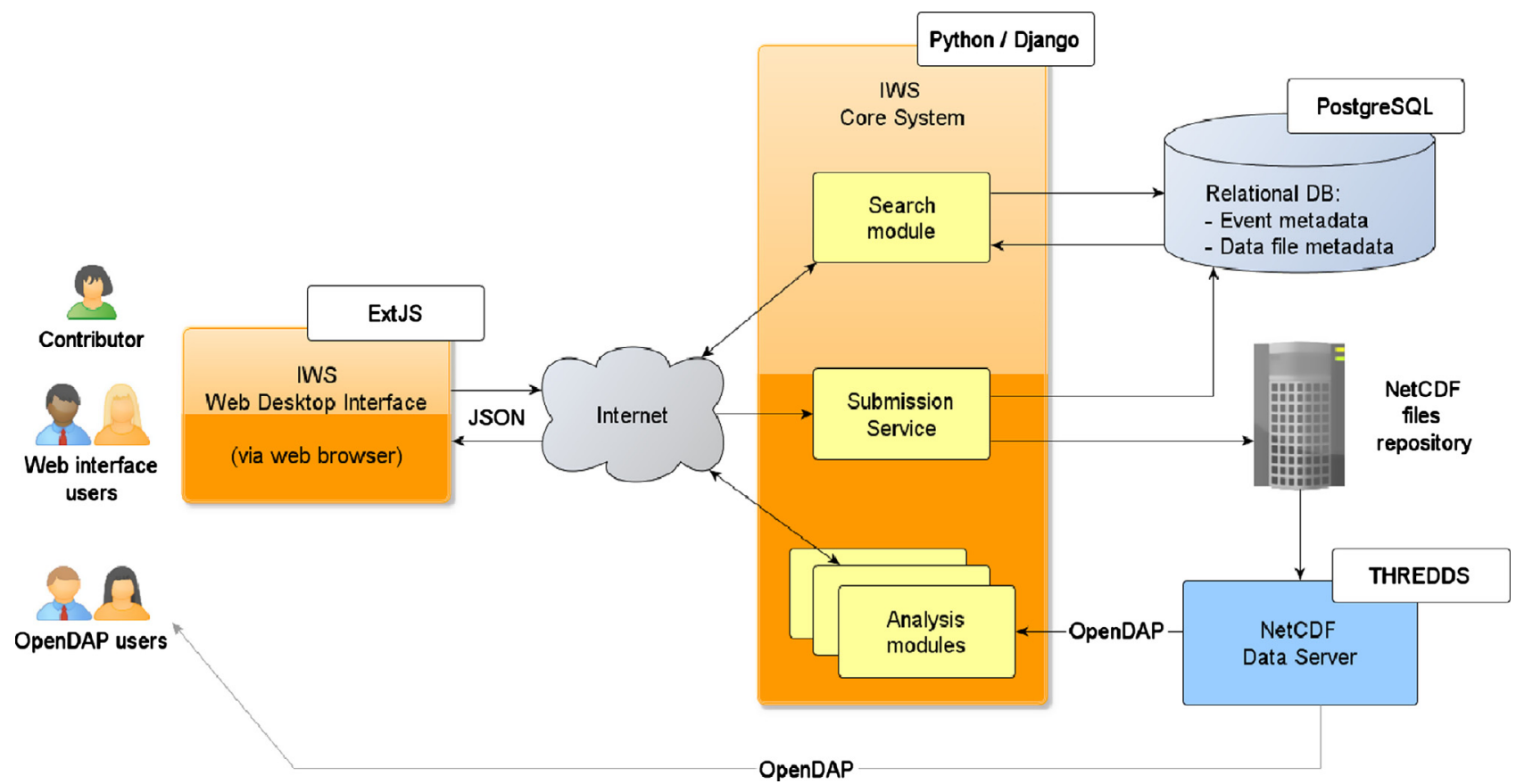

Fig. 24. Architecture design and technical component of the Ionosphere Waves Service system.

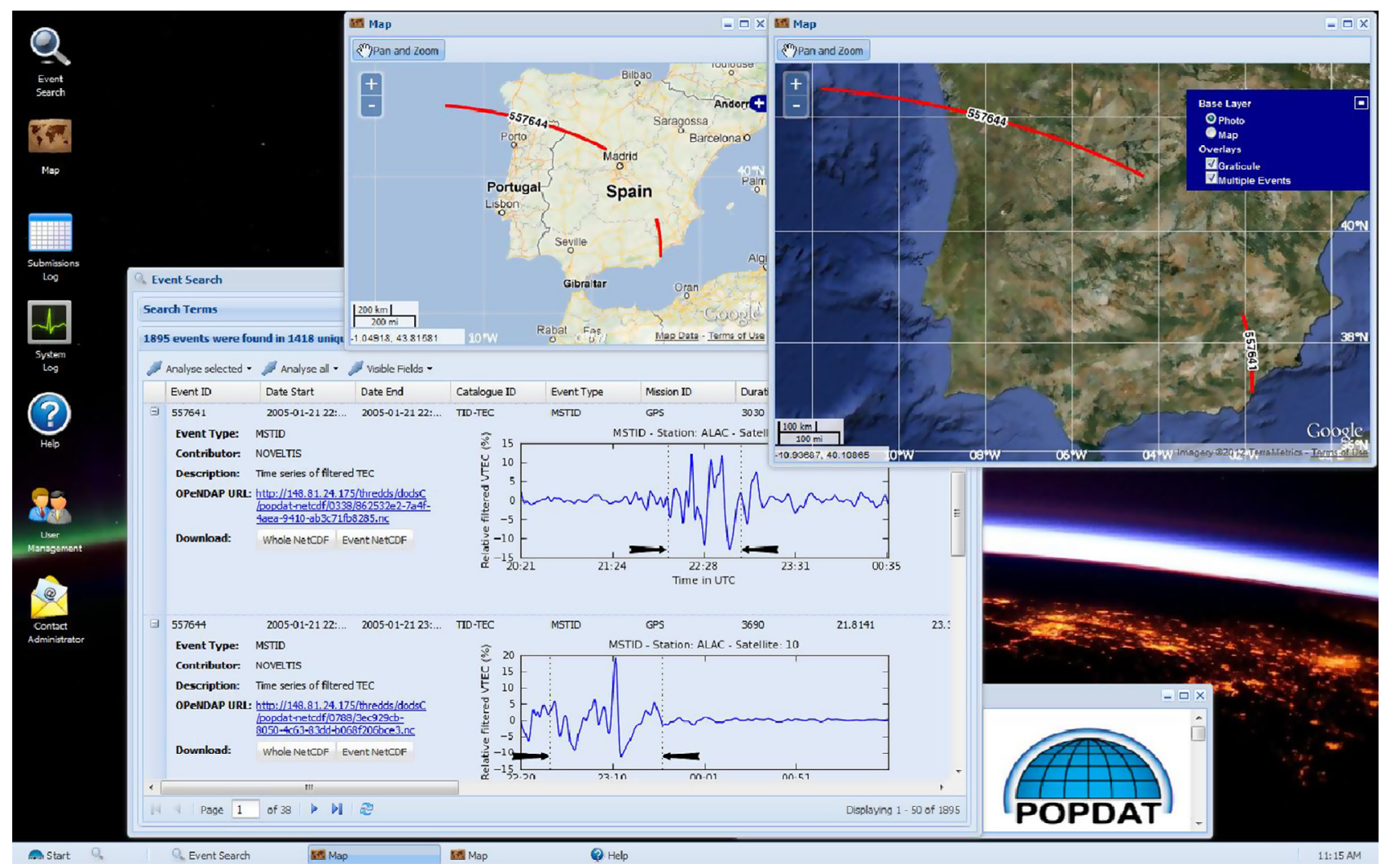

Fig. 25. Web desktop interface of the Ionosphere Waves Service.

- Spiky whistlers (SpW), swallow-tailed whistlers (STW), and X-shaped whistlers between the "normal" and longer path fractional hop whistlers, but without a second step automatic selection.

- Ion whistlers between the short path fractional hop whistlers, but without a second step automatic selection.

The automatic selection of these sub-classes is a further task in the future.
It is important that the application of the adapt bases created by the AWDSat procedure opens the way before the detailed investigations of the connections between different important, related phenomena, such as:

- transient luminous events (TLE's) and whistlers,

- thunderstorms and whistlers,

- seismic activity and whistlers. 

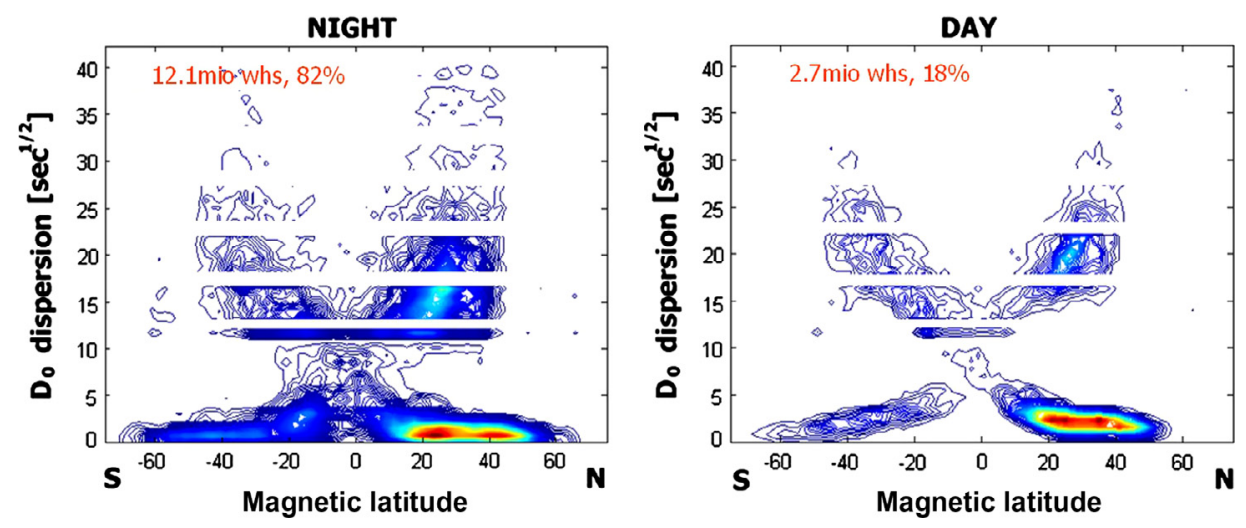

Fig. 26. IWS application example: distribution of dispersion values of trapezoid, short path whistler pairs in the function of the geomagnetic latitude, based on DEMETER records.

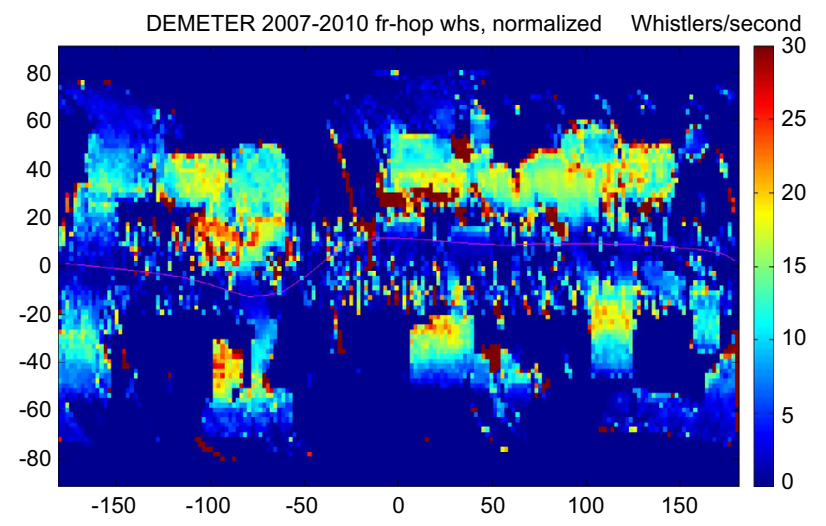

Fig. 27. IWS application example: geographic distribution of the number of fractional hop whistlers (occurrence rates), based on automatic wave recognition in DEMETER data. Note that applied dataset was recorded at sun-syncronous LEO orbit, thus result represents only LT morning and evening periods.

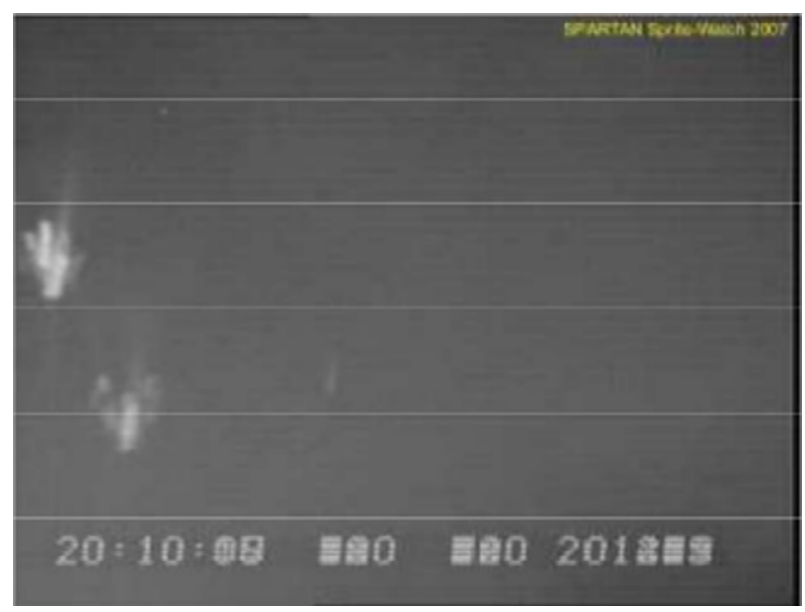

Fig. 28. Picture taken by the camera installed in the observatory at the top of the Sniezka mountain, Poland $\left(50^{\circ} 44^{\prime} \mathrm{N}, 15^{\circ} 44^{\prime} \mathrm{E}\right.$, $1603 \mathrm{~m}$ alt.) on 20th July 2007. Two sprites are clearly seen in this picture. At the time of registration (20:10:08 UT) intense lightning was detected both by the Euclid and Linet systems where the camera was oriented (NW direction, $333^{\circ}$ azimuth, $30^{\circ}$ elevation, courtesy A. Odzimek).

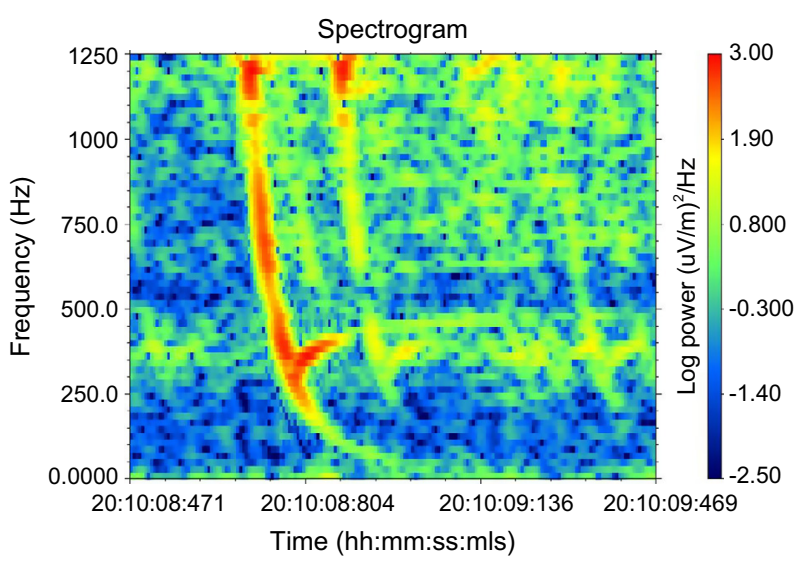

Fig. 29. The dynamic FFT spectrum of fractional hop whistlers recorded simultaneously on board of DEMETER in the ELF band (Błęcki et al. 2009).

\subsubsection{Technique for Atmosphere Gravity Waves (AGW) selection in the satellites measurement data}

The AGW phenomena appear in the onboard records also as a resultant of the total neutral atmosphere density "background" and the AGW transient. However, as a contrast to the electromagnetic transient phenomena the "background" signals in this case are extremely high and "fast changing" comparing to the transient fluctuations in the medium caused by the AGW. Therefore such types of automatic signal processing, which are easily applicable for the electromagnetic whistler-like transients (see section above), do not apply well for AGW phenomena.

The input data source for AGW selection is the archive of Dynamics Explorer 2 mission (DE 2). Eventually we are planning to analyze and display at the Ionosphere Waves Service the data obtained by all satellites of the entire AtmosphereExplorer-satellite-series indicated in Table 1.

DE 2 data are freely available via NASA web-portal (see the National Space Science Data Center at http://nssdc.gsfc.gov/ atmoweb web-portal). The data of the onboard instrument NACS are presented there as tables in ASCII format where each column corresponds to a measured parameter, specifically the individual concentrations of the neutral atmosphere 


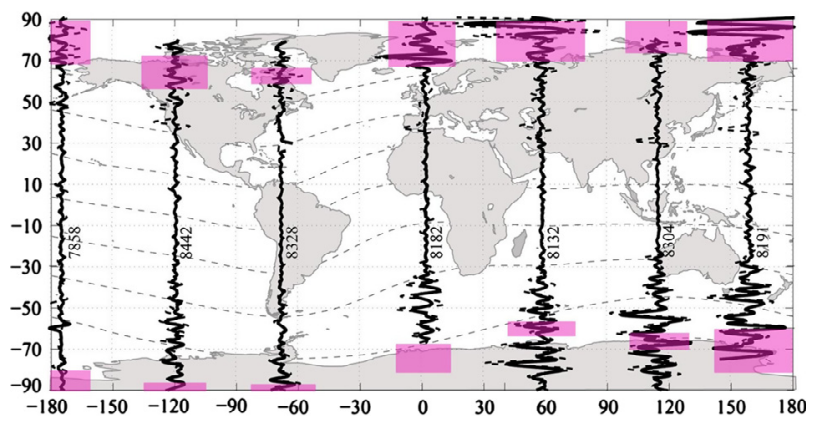

Fig. 30. Global AGW distribution according to direct measurements onboard DE 2 satellite. Waveforms are plotted along the satellite orbits. Current auroral oval positions are highlighted.

components. Also, some accompanying parameters are included such as orbit number, local time, geographical coordinates, etc. The total size of the NACS data is $1.6 \mathrm{~GB}$ and it includes 4644 files with the measurements.

Before the AGW selection procedure the original data were divided into continuous data-blocks without perceptible gaps in $\mathrm{UT},[\mathrm{O}],\left[\mathrm{N}_{2}\right],[\mathrm{He}]$. These blocks are accepted by AGW extraction algorithm as input and then processed individually. As an example, Figure 13 shows gas concentration records in one such block. Corresponding spectra are shown in Figure 14. As can be seen, synchronous small amplitude oscillations of concentrations appear at the background of large-scale trends, which variations are by more than two orders of magnitude greater. The presence of the trend is caused by global latitudinal and altitudinal distributions of atmospheric parameters: the trend is not real oscillatory process, though completely distorts the spectra of initial data. Thus in the original data the AGW spectrum becomes indistinguishable.

Until now, data processing technique used for AGW extraction and analysis was based on the band-pass filtering in the wavelength range of $100-900 \mathrm{~km}$, where the presence of AGW is anticipated (Skorokhod \& Lizunov 2012). In the case of identification of quasi-harmonic variations the filter was tightened to the carrier frequency, as far as it might be possible depending on filter transition region and the length of measurement series. This method of AGW extraction has significant drawback: because of the presence of residual trend in AGWs' spectral band, the large-amplitude AGWs or moderate-amplitude AGWs against the weak trend were only extracted. Other AGWs, though they were seen "by eye" in data tracks, were not substantiated. This made it impossible to build an objective statistical picture of the AGW observations.

During POPDAT project another way of AGW selection was elaborated based on the method of original data series decomposition on separated oscillatory modes using the Hilbert-Huang transform instead of the band-pass filtering.

Hilbert-Huang transformation is a specific technique for signal decomposition to non-orthogonal additive, so-called "internal" modes, some kind of oscillations, without assuming that these variations are strictly periodic. This feature is extremely useful when extracting wave against non-stationary processes. Hilbert-Huang transform is described in papers (Huang et al. 1998; Zhao \& Huang 2001; Rato et al. 2008).

Figure 15 shows the final set of wave modes selected using the Hilbert-Huang transform from experimental data; corresponding spectra are shown in Figure 16. These modes represent physically significant processes: the high frequency noises (most likely of instrumental origin), middle-scale
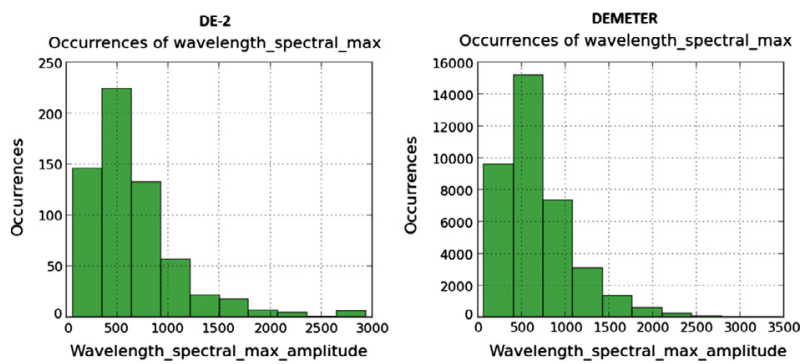

Fig. 31. Statistical estimation of TID wavelength at spectral peakbased on DE 2 and DEMETER data.

AGW, large-scale AGW, and the global scale trend (omitted in the figure). This mining procedure was applied for the all series of DE 2 measurements.

Theoretical criterion verified that selected components of initial signal are really AGW is the fact that phase oscillations of different types of gases depends on their relative molecular weight, in particular, fluctuations in gas concentrations [O] and $[\mathrm{He}]$ have to be going approximately in anti-phase (Makhlouf et al. 1990). The phase shift of obtained wave modes was estimated from their cross-correlation function. For results of sifting shown in Figure 17 the estimation of phase shift gives the value $0.87 \pi$, which agrees well with the AGW theory.

\subsubsection{Technique for Traveling Ionosphere Disturbance (TID) selection in the satellites measurement data}

The problem is similar as in the case of AGWs and the solution has some similarities too.

First and important step in TID recognition over satellite data records is to evaluate the separation boundaries between Polar Cap Convection (PCC) zone and mid and low latitude (MLL) zones in the ionosphere. At mid and low latitudes, bubbles, blobs and/or wave-like structures appear as relatively welldefined signatures along the orbit, relatively small in comparison to the polar cap size. An exception of this assumption, are giant bubble structures, which should be a subject of completely different approach out of the tasks of IWS construction. As an input for the evaluation of the PCC and MLL boundary's position, we use data for horizontal component $V d$ (dotted blue line in Fig. 18) of transverse ion drift velocity fitted by Filtering Cubic Spline (red dot line on bottom panel of Fig. 18). Certain absolute difference $(50-100 \mathrm{~m} / \mathrm{s})$ between horizontal ion drift component and relative zero level from Spline interpolation is assumed to be a sign for Convection Boundary (CB). This procedure is illustrated with $\mathrm{Ni}$, Ti (top panel) and transverse horizontal $V d$ component measured by RPA/IDM instrument onboard Dynamics Explorer-2 satellite (Fig. 14).

In PCC zones: To extract TID structures from the data, we use recently developed Hilbert-Huang Transform (HHT) algorithm (Oya et al. 1986), within convection boundaries (CBCB) for recognition of wave-like signatures in data records. Significant advantage of this signal processing procedure is that it reveals natural frequencies without need of so called relaxation zones at both sides of the data interval. As TID generated at certain latitude could continuously cross $\mathrm{CB}$ in both directions, HHT output wave detection easily connects time series intervals at both sides of the $\mathrm{CB}$. An example of processed data taken in the high latitude part of CB-CB interval already shown in Figures 18 is given in Figure 19. From top to bottom in Figure 19, both HHT of real signal for the Ni (panels a, b) 


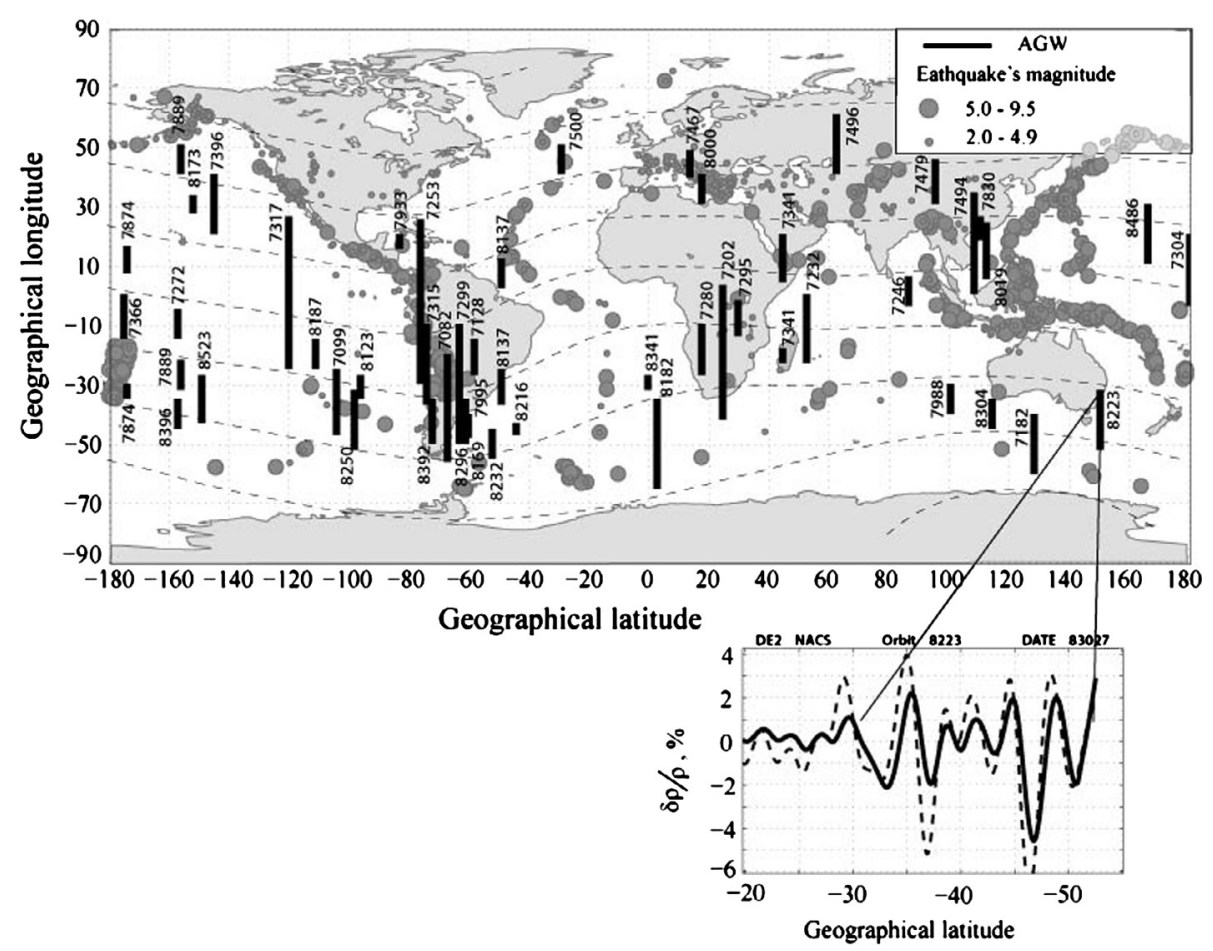

Fig. 32. Geographic distributions of the localized AGW wave packets (solid bars) and epicenters of the earthquakes (circles) occurred during the period November 1982 to February 1983. One of the AGW waveforms is shown in insert.

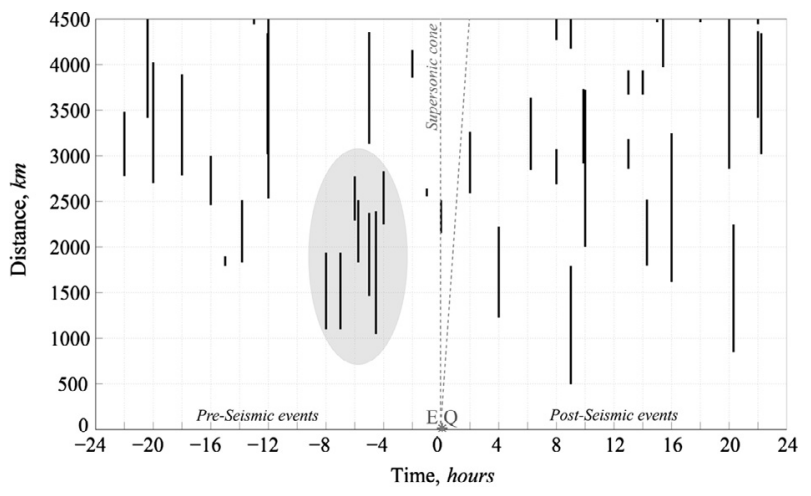

Fig. 33. Causal diagram of coupling between earthquakes (located at the origin of the coordinate system) and localized wave packets of AGW. The group of events at times -8 to $-4 \mathrm{~h}$ is appeared to be earthquake precursors. The "supersonic cone" is highlighted within which no coupling of AGWs and earthquakes is physically possible.

and vertical ion drift $V v$ (panels c, d) are shown. Green lines on panels (b) and (d) correspond to the residual fraction of the signal after HHT processing devoted to average general behavior of data along the orbit. On panels (a) and (c) depict subsequent decomposition of the wave-like structures in $\mathrm{Ni}$ and $V v$ data.

In MML zones: At high latitudes, geomagnetic field could be assumed almost vertical, but toward low latitudes, magnetic inclination changes within $\pm 60^{\circ}-65^{\circ}$ range. From nearly vertical at auroral latitudes, geomagnetic field lines become horizontal at the geomagnetic equator. Due to the limited diffusion across magnetic field lines, ionosphere disturbances generated by AGW in the lower thermosphere, penetrate successfully upward only along the geomagnetic field lines. Thus, depending on the satellite altitude and latitude position, there is a forbidden zone close to the geomagnetic equator, where the effect of TID generated at bottom side F-region could not be observed directly at topside altitudes. Therefore, this limits observational opportunities of in situ satellite techniques for TID recognition in the topside ionosphere at low latitudes. The steps of the TID recognition in these cases are the following: First and important task of the recognition software at MLL is to eliminate effects of such structures ("bubble", "blob", etc. - see e.g. in Oya et al. 1986), which could compromise TID detection by spurious signal generated during data processing. While "bubbles" and "blobs" have asymmetrical behavior, wave-like phenomena have almost symmetrical variations along running median average. Thus, calculating running sum of STD along disturbed ion densities zones, we select and exclude "bubbles" and "blobs" zones from median, as minimum of this sum is used as main indicative parameter for TID recognition. Second, enhanced spectral density at smaller scales is another feature inside "bubbles" and "blobs" structures, which is not typical spectral characteristic for TID. This is an additional opportunity to improve TID recognition which we use as second logical parameter in the software. Third, after TID recognition within ion density disturbance along the orbit, we use HHT to obtain wave-structure and to evaluate main wave amplitude and frequencies.

\subsubsection{GPS Vertical Total Electron Content (VTEC) analysis of Traveling Ionosphere Disturbances (TID)}

As it is well known, the electromagnetic waves propagating through the ionosphere are affected by time delays depending on the signal frequency and electron density. The GPS data produced by bi-frequencies receivers and used by SPECTRE algorithm are respectively for both frequencies $f_{1}(1575.42 \mathrm{MHz})$ and $f_{2}(1227.6 \mathrm{MHz})$ the pseudo-ranges $P_{1}$ and $P_{2}$, and the phase measurements $L_{1}$ and $L_{2}$. By combining these data one 
can compute the slant path delay due to electron content of the ionosphere. The Slant Total Electron Content (STEC), expressed in TEC units, for a receiver satellite couple at epoch $t$ along ray " $i$ " is given by the ionospheric combination:

$$
d_{i}(t)=K\left\{L_{g f}(t)-\left[L_{g f}(t)+P_{g f}(t)\right]\right\}
$$

with

$$
\begin{gathered}
L_{g f}=\lambda_{1} L_{1}-\lambda_{2} L_{2}, \\
P_{g f}=P_{1}-P_{2}, \\
K=\frac{8 \pi^{2} m_{e} \varepsilon_{0}}{e^{2}} \frac{f_{1}^{2} f_{2}^{2}}{\left(f_{1}^{2}-f_{2}^{2}\right)},
\end{gathered}
$$

where $\lambda_{1}, \lambda_{2}$ are the wavelength for $f_{1}$ and $f_{2}, e$ is the charge of one electron, $m_{e}$ is the mass of one electron, and $\varepsilon_{0}$ is the vacuum permittivity. The coefficient $K$ is derived from the secondorder approximation of the refractive index of the ionosphere (see e.g. in Budden 1985). All non-dispersive effects on pseudo-range and phase measurements are avoided by the geometry-free combinations (2) and (3). The averaged sum of these combinations is subtracted to (2) in order to resolve phase ambiguity. (1) is modeled by the sum of STEC and electronic biases affecting GPS satellites, Transmitter Group Delay (TGD), and GPS receivers, Inter-Frequencies Bias (IFB). These biases are assumed constant (Sardón \& Zarraoa 1997). The Slant TEC is redressed to Vertical TEC using the thin shell assumption (Mannucci et al. 1999) with maximum of ionization at $350 \mathrm{~km}$.

$$
\begin{aligned}
& d_{i}(t)=\operatorname{STEC}(t)+\mathrm{IFB}+\mathrm{TGD}, \\
& d_{i}(t)=\frac{\operatorname{VTEC}(t)}{f_{\mathrm{ob}}}+\mathrm{IFB}+\mathrm{TGD},
\end{aligned}
$$

where $f_{\mathrm{ob}}$ is the obliquity factor defined by the thin shell assumption. The intersection of ray $i$ and the thin shell at $350 \mathrm{~km}$ give the position of the observation $d_{i}$ that is usually called the Ionospheric Piercing Point (IPP). In order to assess VTEC maps over GPS network the IPP are interpolated by nearest method on a regular grid. Thus, system of equation (7) is formed and solved by extended Kalman algorithm (Quanrong 1993). Solution provides conjointly an estimation of VTEC on a regular grid and an estimation of the electronic biases IFB and TGD for each receiver and satellite by solving:

$$
d_{1 \ldots N}=G\left[\begin{array}{c}
\operatorname{VTEC}_{1 \ldots P} \\
\mathrm{IFB}_{1 \ldots R} \\
\operatorname{TGD}_{1 \ldots S}
\end{array}\right]
$$

where $N$ is the number of GPS observations, $P$ is the number of grid points, $R$ is the number of GPS receivers, $S$ is the number of GPS satellites, and $G$ is the interpolation operator. The complete logic scheme of the processing chain dedicated to the detection of TIDs from GPS networks is presented in Figure 20.
By the application of these tools mentioned above the production of a useful database for the IWS and the creation of the IWS itself is possible.

\section{The structure of the lonosphere Waves Service - IWS}

The wave extraction methods were carried through effectively on a mass of data, and an easy accessible data set is now ready for scientific investigations. This database contains level 3 data which are comfortable e.g. in Space Weather research. The goal orientation of this level 3 data processing was the extraction of the wave-like phenomena listed above from the raw and firstsecond level processed, i.e. original satellite records. However, to use effectively these level 3 data a complete data service is necessary. This data service is the IWS which is an online tool.

As it was described above the datasets in the IWS are grouped by the main types of phenomena into four topical catalogs:

- AGW catalog for the Atmosphere Gravity Waves (local variation of the neutral density);

- TID catalog for the Traveling Ionosphere Disturbances (in situ, i.e. local variations of the densities of the plasma elements);

- TID-TEC catalog for the variations of the integrated electron density (Total Electron Content) induced by TIDs;

- EMW catalog for the propagating ElectroMagnetic Waves.

By the problem oriented processing of satellite and groundbased data, these catalogs are constructed to provide queriable data fields via Internet-based open access Ionosphere Wave Service. Each catalog comprises specific characteristics of the wave-like structures in the near Earth space as they are extracted from in situ electromagnetic and atmosphere-ionosphere observations. Two major sets of parameters are used to create queriable fields within each Topical catalog, (i) common for all catalogs and (ii) specific for each individual catalog. During data processing, each catalog is uploaded by a set of small size metadata files created for each recognized individual wave event with complete set of above-mentioned queriable parameters that characterize it. In order to maintain real time ability of the Ionosphere Waves Service we apply search opportunities of the system over metadata files relevant for each catalog. This is because it is not feasible that a search be conducted by searching through NetCDF data when the data are as potentially voluminous as it is in IWS. The NetCDF data are of course stored and can be referenced immediately for analysis or extraction, but not for conducting searches.

Therefore, the NetCDF data file exist in IWS archives containing the processed wave data, but also there exists a metadata representation in a relational database allowing fast searches that tells which data file where can be found and what type of event does it contain.

The next principal reason provided in IWS is to provide users with the ability to distinguish between the two cases of:

- an event that was not recorded (no data exists that can be targeted by this query),

- an event that was found not to have occurred (relevant data exists and the query returned showing no event occurred). 
The general architecture of the IWS concept is presented in Figure 21. The Service Topical Catalogs are created and are submitted to the IWS system. These data and metadata sets are the level 3 processed data of the satellite recorded original data and sent to the IWS from the different partners. These level 3 processed data and the created level 3 metadata form the Topical Catalogs and the end users can access the Topical Catalogs via the IWS interface. Therefore it is critical to understand the relationship between Data Files and Events.

Figure 22 is an overview displaying the relationship between the Processed Data and the associated Event Metadata. One of the goals of IWS is to allow scientists to observe an event in one data file and search for the same event in other data files known to exist for the time/space window, as illustrated in Figure 22.

Data files are the processed NetCDF data produced by the POPDAT contributor partners. The files are produced by a process specific for each contributor and data type. Typically they store a number of variables that change with each time step along a satellite orbit track. The exact list of variables is defined for end users in IWS. Events are phenomena detected by the processing algorithm in each Data File, see earlier in Section 4.2. Events are recorded as individual XML files containing specific information for that event, and importantly, all the information that will ever needed to search for that event. The IWS inner data flow is presented in Figure 23.

The architecture design and technical component of the Ionosphere Waves Service system are in Figure 24. In this system for the end users the web interface is the most important. The web interface is constructed in JavaScript using the ExtJS toolkit, providing support for rich UI controls, windows, grids, and other utility functionality. The web interface performs the relatively complex task of helping the user to construct a query to filter out millions of events before displaying and outputting only the data they require. It is expected that the interface will target relatively modern web browsers, for example Google Chrome, Firefox, Opera, Safari, and Internet Explorer 9 and above. The web interface will comprise of several screens for searching and displaying events, wire frame sketches of screens.

The web desktop interface screen is an informative and easy-to-use tool, as it is presented in Figure 25.

In Figure 25 the Event Search window (bottom) is presented with Quick-look of the results and the Map windows (top) display location of results. The application in every case is so simple and informative.

\section{Application demonstrations of the IWS in specific scientific studies}

\subsection{Study on ionospheric upward propagation of whistler-type EM phenomena}

The IWS is intended to open the way for new research directions on complex level. Since the IWS contains mass volume of wave-like events by their metadata system this new database is proven to be an effective tool in the statistical investigations of the event generation and/or appearance. An example of this type of applications is the statistics of the fractional hop whistler signal pairs (trapezoid whistler pairs) identified by AWDSatWHPF software in DEMETER records and the events stored in IWS. In Figure 26 the statistical distribution $D_{0}$ dispersion values of the signal pairs are present as a function of the geomagnetic latitude of recording, composed by IWS yielded whistler parameters of nearly 15 millions of whistler pair occurrences. Detailed characters, e.g. temporal behavior of latitude dependent ionospheric propagation is thus possible to investigate on a statistical base, unlike to low efficient manual data analysis (Hughes \& Rice 2002). In Figure 27 the geographical distribution of automatically detected fractional hop $\left(0^{+}\right)$whistler rates is presented. The IWS-based picture does not exhibit, as expected, strong dependence on regions of known high storm activities (local sources), rather smooth whistler occurrence rate distribution. Note the low whistler rate at the geomagnetic equator.

A more effective investigation of the ionosphere (ionosphere parameters, equatorial anomaly, changing of the whistler occurrence depending on the solar activity, etc.) is possible using the IWS.

\subsection{Application example in the investigation of thunderstorm activity}

Another option in IWS application is e.g. the investigation of the electromagnetic manifestation of thunderstorms. The relation between the thunderstorms and the ionospheric electromagnetic transient activity is also part of the Space Weather research. In this case it is necessary to have simultaneous satellite and ground-based recorded data. The ground-based data (e.g. E-field records, lightning detector network data) sets are not a part of the IWS. However, this example suggests that it will be important in the future to create a new higher level database in which the satellite and ground data will be incorporated. (There was a proposal elaborated in the FP7 dedicated to this topic, but it was not selected.)

Whistlers are electromagnetic waves that can be generated by lightning discharges and are observed in the VLF range. In order to examine most standard way of analysis devoted to examination of electromagnetic manifestation of thunderstorm, DEMETER ICE data, and ground-based registration were utilized. The European example presented here illustrates the general approach of a started investigation. Thunderstorm activity is monitored in Warsaw, where a cluster is built of six antennas. They have been operating since 2009. Each antenna is measuring electric field changes that can be caused by thunderstorms. Discharge events are indicated by the rapid decrease in electric field strength. The limitations of this approach are due to restricted area of ground-based measurements and the nature of the recorded signals. Regional and global lightning location networks, such as EUCLID (euclid.org) and LINET (nowcast.de) in Europe, or WWLLN (wwlln.net), serve vital auxiliary data with lightning locations, time, and intensities.

On the other hand there are satellite electric field registrations. For the purpose of this investigation we present two whistler events that were recorded on DEMETER on 20th July 2007. At the same time SPARTAN Sprite-Watch Team form IMGW Meteorological Observatory located on Śnieżka Mountain in Poland recorded two sprites. Figure 28 presents the two sprites. The distance between the location of the event and the satellite position was around $1200 \mathrm{~km}$. The wave forms were analyzed and as a final output the spectrogram in the ELF and VLF ranges were computed. Figure 29 presents the ELF spectrogram of DEMETER records (Błęcki et al. 2009). Complex analysis of large amount of corresponding events is thus possible utilizing the capabilities of the IWS. This approach 
opens the way for a higher level analysis in Space Weather investigations, and is a strong argument for unified databases containing both ionospheric satellite and ground-based records.

\subsection{IWS as Ionosphere Dynamics Virtual Observatory}

IWS is powerful tool for the visualization and study of the ionosphere dynamics. To demonstrate IWS possibilities let us consider Figure 30, which simply shows a few AGW waveforms taken from IWS database and superimposed on a geographical map. This figure visualizes the global picture of the wave activity of upper atmosphere. It turns out that $\mathrm{AGW}$ wave field has specific morphological structure, including: (1) areas of polar caps, where AGWs are continuously generated and their amplitude reaches maximum values; (2) mid-latitudes, where the intensity of AGWs decreases as the distance from the auroral ovals increases; and (3) latitudes below $40^{\circ}-50^{\circ}$, where the wave activity reaches minimum but does not fully disappear. Moreover, on this global wave back-ground the isolated wave bursts (distinctively localized wave packets) are sporadically observed.

The next Figure 31 represents result of the application of IWS search opportunities to TID-catalog. This visualization provided by ordinary IWS' tool proves that, contrary to popular belief, there is no real physical border between middle-scale and large-scale TIDs, this common division is only conventional.

\subsection{Statistical study of the seismo-ionosphere disturbances}

Here we develop an approach originally proposed in the work (Skorokhod \& Lizunov 2012). Let us analyze possible relation of earthquakes to generation of localized wave packets of AGW (denoted in the AGW catalog as "LAGW"). We would be only interested in the evidences of correlation between seismic and ionospheric events, regardless of any hypothetical mechanisms of seismo-ionospheric coupling. Figure 32 shows geographical distribution of the earthquakes occurred during the period 1st November 1982-15th February 1983 together with locations of LAGWs detected by DE 2 satellite. This figure suggests that ionosphere disturbances and earthquakes are somehow connected (especially, if one takes into account that, during the propagation, AGW shifts from the source horizontally over thousands of kilometers).

Further, let's restrict the set of earthquakes that can serve as sources of LAGWs by intuitive criteria: (1) earthquakes are strong with magnitudes of $M>5.0$, (2) earthquakes are shallow focus with hypocenter depths $D<100 \mathrm{~km}$. Figure 33 shows a causal diagram where all earthquakes selected in such a way are placed in the reference origin using the method of superimposed epochs and the LAGWs are shown with respect to them in the time-distance coordinate system. If time is negative, the LAGWs may appear as precursors of earthquakes; if time is positive, they may be responses to earthquakes. A supersonic cone is shown within which no coupling is physically possible. This figure distinguishes a group of AGWs at times from -8 to $-4 \mathrm{~h}$ and distances from 1000 to $2500 \mathrm{~km}$ around the epicenters as earthquake precursors.

\section{Summary}

The Ionosphere Waves Service, which is in operation, is an excellent tool in the different field of the Space Weather, earthquake, and ionosphere dynamics research. It opens new possibilities in space research and Earth sciences with the unification of data sets recorded by different satellites.

In the future the creation a new high level database containing the IWS and different ground recorded data sets is an important task in increasing the effectiveness of research in Space Weather and other Earth sciences.

Acknowledgements. This work was made with the support of EU FP7 Grant \# 263240, in the POPDAT project. Authors are grateful to the Guest Investigator program issued by CNES for the DEMETER mission for supplying the raw data.

\section{References}

Afraimovich, E.L., E.A. Kosogorov, L.A. Leonovich, K.S. Palamartchouk, N.P. Perevalova, and O.M. Pirog, Observation of large-scale traveling ionospheric disturbances of auroral origin by global GPS networks, Earth Planets Space, 52, 669-674, 2000 .

Afraimovich, E.L., E.A. Kosogorov, L.A. Leonovich, O.S. Lesyuta, and I.I. Ushakov, GPS detection of the instantaneous response of the global ionosphere to strong magnetic storms with sudden commencement, Ann. Geophys., 45 (1), 41-53, 2002.

Afraimovich, E.L., I.K. Edemskiy, S.V. Voeykov, Yu.V. Yasyukevich, and I.V. Zhivetiev, The First GPS-TEC imaging of the space structure of MS wave packets excited by the solar terminator, Ann. Geophys., 27, 1521-1525, 2009.

Artru, J., V. Ducic, H. Kanamori, P. Lognonne, and M. Murakami, Ionospheric detection of gravity waves induces by tsunamis, Geophys. J. Int., 160, 840-848, DOI: 10.1111/j.1365-246X.2005.02552.x, 2005.

Balthazor, R.L., and R.J. Mofett, A study of atmospheric gravity waves and travelling ionospheric disturbances at equatorial latitudes, Ann. Geophys., 15, 1048-1056, 1997.

Błęcki, J., M. Parrot, and R. Wronowski, ELF and VLF signatures of sprites registered onboard the low altitude satellite DEMETER, Ann. Geophys., 27, 2599-2605, 2009.

Berthelier, J.-J., M. Godefroy, F. Leblanc, E. Seran, D. Peschard, P. Gilbert, and J. Artru, IAP, the thermal plasma analyzer on DEMETER, Planet. Space Sci., 54, 487-501, 2006.

Budden, K.G., The propagation of radio waves: The theory of radio waves of low power in the ionosphere and magnetosphere, Cambridge University Press, Cambridge and New York, 1985.

Cai, H.T., F. Yin, S.Y. Ma, and I.W. McCrea, Observations of AGW/ TID propagation across the polar cap: a case study, Ann. Geophys., 29, 1355-1363, 2011.

Calais, E., and J.S. Haase, Detection of ionospheric perturbations using a dense GPS array in Southern California, Geophys. Res. Lett., 30 (12), 1628, DOI: 10.1029/2003GL017708, 2003.

Chum, J., F. Jiricek, O. Santolik, M. Parrot, G. Diendorfer, and J. Fiser, Assigning the causative lightning to the whistlers observed on satellites, Ann. Geophys., 24, 2921-2929, 2006.

Collier, A. B., J. Lichtenberger, M. A. Clilverd, P. Steinbach, and C. J. Rodger, Source region for whistlers detected at Rothera, Antarctica, J. Geophys. Res., 116, A03219, DOI: 10.1029/2010JA016197, 2011.

Crespon, F., Tomographie 2D et 3D de l'ionosphère par GPS: applications aux aléas géophysiques. Thesis, Institue de Physique du Globe de Paris, 2007.

Crespon, F., E. Jeansou, J. Helbert, G. Moreaux, P. Lognonné, P.E. Godet, and R. Garcia, SPECTRE (www.noveltis.fr/spectre): a web Service for Ionospheric Products, in Proceedings of 1st Colloquium Scientific and Fundamental Aspects of the Galileo Programme, Toulouse, France, October, 2007.

Fedorenko, A.K., G.V. Lizunov, and H. Rothkaehl, Satellite observations of wavelike atmosphere perturbations caused by strong earthquakes, Geomag. Aeron., 45, 403-410, 2005.

Ferencz, Cs., O.E. Ferencz, D. Hamar, and J. Lichtenberger, Whistler phenomena, short impulse propagation, Kluwer Academic Publishers, Dordrecht, 2001. 
Ferencz, O.E., Cs. Ferencz, P. Steinbach, J. Lichtenberger, D. Hamar, M. Parrot, F. Lefeuvre, and J.-J. Berthelier, The effect of subionospheric propagation on whistlers recorded by the DEMETER satellite - observation and modeling, Ann. Geophys., 25, 1103-1112, 2007.

Ferencz, O.E., L. Bodnár, Cs. Ferencz, D. Hamar, J. Lichtenberger, P. Steinbach, V. Korepanov, G. Mikhaylova, Yu. Mikhaylov, and V. Kuznetsov, Ducted whistlers propagating in higher order guided mode and recorded on board of Compass- 2 satellite by the advanced Signal Analyzer and Sampler SAS2, J. Geophys. Res., 114, A03213, DOI: 10.1029/2008JA013542, 2009.

Ferencz, Cs., J. Lichtenberger, D. Hamar, O.E. Ferencz, P. Steinbach, B. Székely, M. Parrot, F. Lefeuvre, J.-J. Bertelier, and M.A. Clilverd, An unusual VLF signature structure recorded by the DEMETER satellite, J. Geophys. Res., 115, A02210, DOI: 10.1029/2009JA014636, 2010.

Francis, S.H., Global propagation of atmospheric gravity waves: a review, J. Atmos. Terr. Phys., 37, 1011-1054, 1975.

Golden, D.I., M. Spasojevic, and U.S. Inan, Determination of solar cycle variations of midlatitude ELF/VLF chorus and hiss via automated signal detection, J. Geophys. Res., 116, A03225, DOI: 10.1029/2010JA016193, 2011.

Hayashi, H., N. Nishitani, T. Ogawa, Y. Otsuka, T. Tsugawa, K. Hosokawa, and A. Saito, Large-scale traveling ionospheric disturbance observed by superDARN Hokkaido HF radar and GPS networks on 15 December 2006, J. Geophys. Res., 115, A06309, DOI: 10.1029/2009JA014297, 2010.

Helliwell, R.A., Whistlers and related ionospheric phenomena, Stanford University Press, Stanford, 1965.

Hines, C.O., Internal atmospheric gravity waves at ionospheric heights, Can. J. Phys. 38, 1441-1481, 1960

Hines, C.O., The upper atmosphere in motion, in Geophysical Monograph, vol. 18, American Geophysical Union, Washington D.C, 1974 .

Hocke, K., and K. Schlegel, A review of atmospheric gravity waves and traveling ionospheric disturbances: 1982-1995, Ann. Geophys., 14, 917-940, 1996.

Huang, N.E, Z. Shen, S.R. Long, M.C. Wu, H.H. Shih, Q. Zheng, N-C. Yen, C.C. Tung, and H.H. Liu, The empirical mode decomposition and the Hilbert spectrum for nonlinear and non-stationary time series analysis, Proc. R. Soc. London, 454 903-995, DOI: 10.1098/rspa.1998.0193, 1998.

Hughes, A.R.W., and W.K.M. Rice, A satellite study of low latitude electron and proton whistlers, J. Atmos. Sol. Terr. Phys., 59 (10), 1217-1222, 1997.

Hughes, A.R.W., and W.K.M. Rice, The latitudinal dependence of whistler 'ghost' delay times, Adv. Space Res., 30, 2619-2624, 2002.

Inan, U.S., D. Piddyachiy, W.B. Peter, J.A. Sauvaud, and M. Parrot, DEMETER satellite observations of lightning-induced electron precipitation, Geophys. Res. Lett., 34, L07103, DOI: 10.1029/2006GL029238, 2007.

Kato, S., Developments of the Earth and planetary sciences, in Dynamics of the upper atmosphere, Center for Academic Publications, Japan/Tokyo, 1980.

Lefeuvre, F., R. Marshall, J.L. Pincon, U.S. Inan, D. Lagoutte, M. Parrot, and J.-J. Berthelier, On remote sensing of transient luminous events' parent lightning discharges by ELF/VLF wave measurements on boar a satellite, J. Geophys. Res., 114, A09303, DOI: 10.1029/2009JA014154, 2009.

Lichtenberger, J., Cs. Ferencz, L. Bodnár, D. Hamar, and P. Steinbach, Automatic Whistler Detector and Analyzer system: Automatic Whistler Detector, J. Geophys. Res., 113, A12201, DOI: 10.1029/2008JA013467, 2008.
Lichtenberger, J., Cs. Ferencz, D. Hamar, P. Steinbach, C.J. Rodger, M.A. Clilverd, and A.B. Collier, Automatic Whistler Detector and Analyzer system: Implementation of the analyzer algorithm, J. Geophys. Res., 115, A12214, DOI: 10.1029/2010JA015931, 2010.

Lognonné, P., J. Artru, R. Garcia, F. Crespon, V. Ducic, E. Jeansou, O. Occhipinti, J. Helbert, G. Moreaux, and P.E. Godet, Ground based GPS imaging of ionospheric post-seismic signal, Planet. Space Sci., 54, 528-540, 2006.

Makela, J. J., P. Lognonné, H. Hébert, T. Gehrels, L. Rolland, et al., Imaging and modeling the ionospheric airglow response over Hawaii to the tsunami generated by the Tohoku earthquake of 11 March 2011, Geophys. Res. Lett., 38, L00G02, DOI: 10.1029/2011GL047860, 2011.

Makhlouf, U., E. Dewan, J.R. Isler, and T.F. Tuan, On the importance of the purely gravitationally induced density, pressure and temperature variations in gravity waves: Their application to airglow observations, J. Geophys. Res., 95, 4103-4111, 1990.

Mannucci, A.J., B. Iijima, L. Sparks, X. Pi, B. Wilson, and U. Lindqwister, Assessment of global TEC mapping using a threedimensional electron density model, J. Atmos. Terr. Phys., 61, 1227-1236, 1999

Meredith, N.P., R.B. Horne, M.A. Clilverd, D. Horsfall, R.M. Thorne, and R.R. Anderson, Origins of plasmaspheric hiss, $J$. Geophys. Res., 111, A09217, DOI: 10.1029/2006JA011707, 2006.

Mayer, H.G., I. Harris, F.A. Herrero, N.W. Spencer, F. Varosi, and W.D. Pesnell, Thermospheric gravity waves: observations and interpretation using the transfer function model (TFM), Space Sci. Rev., 54, 297-375, 1990

Nemec, F., O. Santolik, M. Parrot, and C.J. Rodger, Relationship between median intensities of electromagnetic emissions in the VLF range and lightning activity, J. Geophys. Res., 115, A08315, DOI: 10.1029/2010JA015296, 2010.

Nunn, D., A. Demekhov, V. Trakhtengerts, and M.J. Rycroft, VLF emission triggering by a highly anisotropic energetic electron plasma, Ann. Geophys., 21, 481-492, 2003.

Oya, H., T. Takahashi, and S. Watanabe, Observation of low latitude ionosphere by the impedance probe on board the Hinotori satellite, J. Geomag. Geoelectr., 38, 111-123, 1986.

Quanrong, C., Approximate Kalman filtering, in Series in approximations and decompositions, vol. 2, World Scientific, Singapore, 1993.

Parrot, M., U.S. Inan, and N.G. Lehtinen, V-shaped VLF streaks recorded on DEMETER above powerful thunderstorms, J. Geophys. Res., 113, A10310, DOI: 10.1029/2008JA013336, 2008.

Parrot, M., U.S. Inan, N.G. Lehtinen, and J.L. Pincon, Penetration of lightning MF signals to the upper ionosphere over VLF groundbased transmitters, J. Geophys. Res., 114, A12318, DOI: 10.1029/2010JA015296, 2009.

Rato, R.T., M.D. Ortigueira, and A.G. Batista, On the HHT, its problems, and some solutions, Mechanical Systems and Signal Processing, 22, 1374-1394, 2008.

Sardón, E., and N. Zarraoa, Estimation of total electron content using GPS data: How stable are the differential satellite and receiver instrumental biases? Radio Science, 32, 1899-1910, 1997.

Skorokhod, T., and G. Lizunov, The localized acoustic gravity wave packets in the ionosphere, Geomag. Aeron., 52 (1), 88-93, 2012.

Steinbach, P., Investigation of upper-atmospheric ionization processes using ground based and satellite VLF recordings. PhD Thesis, Eötvös University, Budapest (in Hungarian), 2006.

Zhao, J., and D. Huang, Mirror extending and circular spline function for empirical mode decomposition method, Journal of Zhejiang University (Science), 2 (3), 247-252, 2001.

Cite this article as: Ferencz C, Lizunov G, Crespon F, Price I, Bankov L, et al.: Ionosphere Waves Service (IWS) - a problem-oriented tool in ionosphere and Space Weather research produced by POPDAT project. J. Space Weather Space Clim., 2014,4 , A17. 